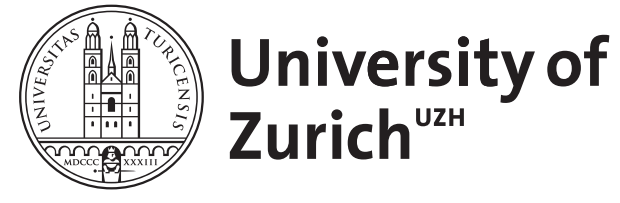
Archive

University of Zurich

University Library

Strickhofstrasse 39

CH-8057 Zurich

www.zora.uzh.ch

Year: 2011

Schreibweise des Seins? Zur Verwendung der Auslassungspunkte auf der Suche nach einer Sprache des Erlebens

Abbt, Christine

Posted at the Zurich Open Repository and Archive, University of Zurich

ZORA URL: https://doi.org/10.5167/uzh-49983

Book Section

Published Version

Originally published at:

Abbt, Christine (2011). Schreibweise des Seins? Zur Verwendung der Auslassungspunkte auf der Suche nach einer Sprache des Erlebens. In: Giertler, M; Köppel, R. Von Lettern und Lücken. Zur Ordnung der Schrift in Bleisatz. München: Fink, 129-161. 


\section{Schreibweise des Seins? Zur Verwendung der Auslassungspunkte auf der Suche nach einer Sprache des Erlebens}

Die Suche nach einem adäquaten sprachlichen Ausdruck für das Erleben eint sehr unterschiedliche literarische und philosophische Ansätze. Dementsprechend werden innerhalb der Geschichte der Literatur und Philosophie diverse Varianten erprobt, um sprachlich zugänglich zu machen, was sich der direkten Vermittlung widersetzt. Ab dem ausgehenden 18. Jahrhundert und noch bis in die Spätmoderne kann der Einsatz von Auslassungspunkten als eine solche Variante begriffen werden. Vom Platzhalter für ausgespartes Sprachmaterial wandelt sich das Auslassungszeichen in manchen Texten zum Kronzeugen jener Abwesenheit, die das moderne Sprachverständnis zentriert. Die drei Punkte transformieren dabei vom traditionellen Satzzeichen zu einer Schreibweise, welche für die Auffassung agiert, dass die Grenze der Sprache nicht zusammenfällt mit der Grenze des Erlebens.

Es ist diese Eigenschaft, die die Verwendung der drei Punkte in Texten der Postmoderne fragwürdig werden lässt. Die Punktspuren werden dort durch Strichlein ersetzt, die statt den Dimensionen von Raum und Konstellation das Moment von Zeit und Nachträglichkeit gewichten. An Beispielen der modernen und postmodernen Literatur und Philosophie wird im Folgenden dem Zeichen für Ausgelassenes bzw. für das Auslassen nachgegangen. Die mögliche Geschichte der Schreibweise, die Roland Barthes beginnen lässt, wird dabei in Auseinandersetzung mit der Verwendung eines einzelnen Satzzeichens, den drei Punkten, fortgesetzt.

\section{Die Punkte als eine Schreibweise}

Als eine „Schreibweise“ par excellence nennt Roland Barthes in seiner Schrift Am Nullpunkt der Literatur ${ }^{1}$ Jacques Héberts ${ }^{2}$ Verwendung von Kraftausdrücken in den Ausgaben des Père Duchêne. Diese wiederkehrenden Grobheiten sagen an sich nichts und doch kommt ihnen eine spezifische Funktion zu. Barthes bezeichnet

1 Roland Barthes, Am Nullpunkt der Literatur, übers. v. Helmut Scheffel, Frankfurt am Main, 1982.

2 Hébert war Politiker und Herausgeber des radikalrevolutionären Père Duchêne, als Mitglied der Pariser Commune wurde er 1794 guillotiniert. 
sie als Signale, die hier eine insgesamt revolutionäre Situation bedeuten: „Wir haben hier das Musterbeispiel einer Schreibweise, die nicht mehr nur etwas mitteilen oder ausdrücken, sondern darüber hinaus ein außerhalb des Mitgeteilten Liegendes bedeuten will, das zugleich das geschichtliche Geschehen ist und der Anteil, den man daran nimmt. ${ }^{\text {" }}$ Die Schreibweise umfasst eine im Schriftbild auszumachende formale Gegebenheit, die nicht nur etwas mitteilt oder ausdrückt, sondern darüber hinaus eine historische Situation zeigt und jene Position, die der Textproduzent innerhalb dieser Situation einnimmt. Neben Sprache und Stil bestimmt Barthes die Schreibweise als eine dritte Dimension der Form, die jede literarische Schrift zur Schau stellt und einmal als Moral der sprachlichen Ausdrucksweise ${ }^{4}$, einmal als Ethos bezeichnet wird: „In jeder beliebigen literarischen Form findet sich die allgemeine Wahl eines Tones, oder wenn man so will: eines Ethos, und hier individualisiert sich ein Schriftsteller eindeutig, denn hier engagiert er sich. ${ }^{“ 5}$ Die formale Dimension der Schreibweise, die Barthes interessiert und die er in der Folge in seiner Schrift für die literarische Moderne zu beschreiben beabsichtigt, unterscheidet sich von der allgemeinen Sprache und vom Stil eines Einzelnen. Die dritte Dimension der Form hat eine spezifische Funktion innerhalb eines Textganzen. Sie markiert vor einem historischen Hintergrund eine Parteinahme. Ob diese formale Wirklichkeit, wie Barthes vorschlägt, in jeder literarischen Schrift auszumachen ist, steht hier nicht zur Frage. Aber ob es sich bei den gehäuften Punktspuren in modernen Texten um eine Dimension von Form handelt, die als Schreibweise begriffen werden kann und zwar als eine Schreibweise des Seins, diese Frage wird im Folgenden weiter erörtert.

\section{Vom Satzzeichen zum Signal}

Die grammatikalische Geschichte des Auslassungszeichens beginnt bei der Abkürzung eines Wortes, beim Fehlen eines Namens oder der Streichung eines Satzteiles. Die Punkte (oder Sternchen oder Striche) stehen dabei als Ersatz für etwas Bestimmtes. ${ }^{6}$ Bis heute entspricht dies der einen sprachlichen Funktion der Auslassungspunkte. Diese fungieren dabei als Statthalter für bewusst Weggelassenes oder noch Fehlendes und ökonomisieren. Der Text wird kürzer, die zitierte Aussage wird auf das Wesentliche reduziert, die Effizienz der Lektüre wird gefördert, problematische Passagen werden ausgespart. Bereits hier kommt den Punkten eine Funktion zu.

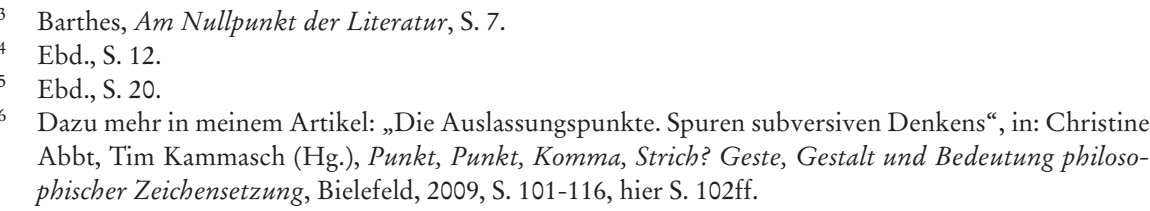


Signale im Sinne von Barthes werden die Punkte dort, wo sie ein besonderes Sprachbewusstsein demonstrieren, dafür Partei ergreifen. Dass Adorno in seinen Noten zur Literatur auf kein anderes Zeichen so ablehnend reagiert wie auf die Setzung der drei Punkte, könnte durchaus schon als Indiz gelten, dass sich spätestens Ende des 19. Jahrhunderts die Transformation des traditionellen Satzzeichens zu einer Schreibweise vollzogen hat: „Die drei Punkte, mit denen man in der Zeit des zur Stimmung kommerzialisierten Impressionismus Sätze bedeutungsvoll offen zu lassen liebte, suggerieren die Unendlichkeit von Gedanken und Assoziationen, die eben der Schmock nicht hat, der sich darauf verlassen muss, durchs Schriftbild sie vorzuspiegeln. Reduziert man aber, wie die Georgeschule, jene den unendlichen Dezimalbrüchen der Arithmetik entwendeten Punkte auf die Zahl zwei, so meint man, die fiktive Unendlichkeit ungestraft weiter beanspruchen zu können, indem man, was dem eigenen Sinn nach unexakt sein will, als exakt drapiert. Der Interpunktion des unverschämten Schmocks ist die des verschämten nicht überlegen. “7 Die Punkte werden zum Gegenstand von Kritik, weil sie als Schreibweise die Unendlichkeit von Gedanken und Assoziationen inszenieren und damit eine bestimmte Auffassung von Sprache bejahen.

Der Wandel vom traditionellen Satzzeichen zum Signal setzt allerdings bereits früher ein. Johann Bodmer beschreibt 1768 in seinen Grundsätzen der deutschen Sprache den Gebrauch der Auslassungszeichen folgendermassen: „Dem Leser mehr zu denken geben als man sagt [...]. ${ }^{\text {" }}$ Die Auslassungspunkte werden hier zum ersten Mal explizit als Operatoren des selbständigen Weiterdenkens vorgestellt. Wo sie gesetzt sind, werden die Lesenden zur Interpretation und individuellen Fortschreibung herausgefordert. Die Punkte lösen sich damit aus ihrem eindeutigen Stellvertreterdasein. Sie sind gezielte Einladung zum Selbstdenken und strukturieren die Rezeptionstätigkeit des Lesers, der Leserin als eine aktive und eigenständige. Vielleicht nicht zufällig taucht der Begriff der ,Stimmung' zum ersten Mal nur wenige Jahre später, nämlich 1776 in Goethes „Falconet“-Aufsatz auf. ${ }^{9}$ Die zeitliche Koinzidenz des neuen Verständnisses von Punkten, die den Lesenden animieren sollen, den eigenen Erfahrungshorizont einzubringen, und dem neu verwendeten Begriff der Stimmung, der bei Goethe im Zusammenhang mit einer Qualität gedacht wird, welche der Künstler erspüren und in die Objektivität eines Ausdrucks umformen soll, ist kaum zufällig.

Denis Diderots eigenwilliger Roman Jacques le Fataliste, der in Frankreich 1796 erscheint, ${ }^{10}$ ist auffällig angereichert mit Auslassungspunkten. Die Punkte sind

7 Theodor W. Adorno, „Satzzeichen“, in: Ders., Noten zur Literatur I, Frankfurt am Main, 2003, S. 106-113, hier S. 109.

8 Johann J. Bodmer zitiert nach Stefan Höchli, Zur Geschichte der Interpunktion im Deutschen, Berlin, New York, 1981, S. 225.

9 David E. Wellbery, „Stimmung“, in: Karlheinz Barck u.a. (Hg.), Historisches Wörterbuch ästhetischer Grundbegriffe, Bd. 5, Stuttgart, Weimar, 2003, S. 703-733.

10 Die genaue Entstehungszeit des Romans ist unbekannt. Zuerst hat er sich durch Abschriften verbreitet. Goethe hat eine solche bereits 1780 gelesen und soll davon begeistert gewesen sein. 
in Diderots Roman ein formales Element, womit die strenge Schriftsprache zum Beispiel eines Voltaires durchbrochen und kritisiert und durch eine lebendige, alltagsnahe Ausdrucksweise ersetzt wird. Die Punkte führen die Schrift zurück zum gesprochenen Wort. Von Beginn weg tauchen in Diderots Text die Punkte sowohl in den Passagen der Dialoge zwischen Jacques und seinem Herrn auf als auch in der Rede des Erzählers. Noch bevor der Inhalt jeder Passage im Einzelnen erfasst wird, trägt das Schriftbild eine Geste von Kritik vor. Das Ideal eines in sich völlig geschlossen durchkomponierten Textkorpus wird schriftbildlich aufgebrochen, noch bevor diese Absicht auch durch den Gehalt der Sprache bekräftigt wird. Diese frühen Verweise auf die Auslassungspunkte als Signale für die Suche nach einer neuen Sprache und ihre Verbindung mit der Vermittlung von lebendigen Erlebensqualitäten leiten ein, was, wie Adorno festhält, im 19. Jahrhundert erst richtig in Mode kommt und noch bis ins 20. Jahrhundert hinein wirksam bleibt. ${ }^{11}$

Barthes weist darauf hin, dass Schreibweisen zwar stets nur vor dem Horizont geschichtlicher Situationen zu begreifen sind, diese aber gleichsam Autoren verbinden können, die Jahrzehnte oder Jahrhunderte auseinander liegen und solche trennt, die in derselben Zeit schreiben. Barthes führt als Beispiele Mérimée und Fénélon an, deren Schreibweisen sich gleichen, obwohl eineinhalb Jahrhunderte sie trennen: „Im Gegensatz dazu verwenden fast gleichzeitig lebende Autoren wie Mérimée und Lautréament, Mallarmé und Céline, Gide und Queneau, Claudel und Camus, Autoren, für die der gleiche historische Zustand unserer Sprache gegeben ist, Schreibweisen, die sich zutiefst voneinander unterscheiden. Alles trennt sie: der Ton, der Vortrag, der Zweck, die Moral, das Naturell ihres Sprechens, so daß die Gemeinsamkeit der Epoche und der Sprache sehr wenig bedeutet gegenüber den so verschiedenen Schreibweisen, die sich wiederum gerade durch ihre Gegensätzlichkeit so klar definieren." ${ }^{2}$ Dass in Bezug auf die Verwendung der Punkte Diderot mit Céline zu vergleichen ist ebenso wie Schnitzler mit Bernhard, obwohl die Zeit sie trennt, ist später weiter auszuführen.

\section{Die Suche nach einer Sprache des Erlebens}

Das Aufkommen der Punkte in neuer Setzung fällt zeitlich zusammen mit der Herausbildung der Ansprüche der Moderne. Bis Mitte des 19. Jahrhunderts differenziert sich das Profil so aus wie es noch bis weit ins 20. Jahrhundert wirksam bleibt: „Die gesamte Literatur von Flaubert bis heute ist zu einer Problematik der Sprache geworden. ${ }^{\text {13 }}$ Die einheitliche Schreibweise von Klassik und Romantik,

11 Zur früheren Verwendung der Punkte in der russischen Literatur: Brigitte Obermayr, „Auslassungspunkte als Materialspur am Beispiel von A. S. Puskins ,Evgenji Onegin““, in: Sonderforschungsbereich 626 (Hg.), Ästhetische Erfahrung. Gegenstände, Konzepte, Geschichtlichkeit, Berlin, 2006.

12 Barthes, Am Nullpunkt der Literatur, S. 21.

13 Ebd., S. 9. 
die von einem idealen Zusammenspiel zeugt zwischen einem universalen Geist und immateriellen Zeichen, die sich ohne eigene Wirkkraft an den Geist verausgaben, weicht einer Schreibweise, so die Ausführung von Barthes, die die Schwierigkeit von Sprache inszeniert und involviert. Die Literatur wird in diesem Wandlungsgeschehen zum Objekt. Im Unterschied zur klassischen Literatur, die Sprache war, konstituiert sich die neue Literatur als Ausdrucksform. Sie bekräftigt ihre Rolle als Vermittlerin: Sie wird zu einer Art Philosophie mit anderen Mitteln. Die literarische Form dient nicht mehr nur „der Ökonomie oder der Euphemie“. Sie entfaltet ein eigenes Gewicht und wird wahrgenommen als „tiefe Sprache voller Geheimnisse ${ }^{“ 14}$. Die Folgen der neuen Paradigmen sind gravierend: „Die literarische Form kann von nun an jene existentiellen Gefühle erwecken, die mit der Hohlseite jedes Objektes verbunden sind: das Bedeuten des Ungewöhnlichen [...]. ${ }^{“ 15}$ Die Schreibweise der modernen Literatur öffnet sich damit sowohl gegenüber dem Produktions- als auch dem Rezeptionsprozess. Das Bewusstsein des Autors sucht nach einer passenden Form, die Leserin sucht nach einer Deutung des Zeichenmaterials. Die Schrift wird dabei zum Medium, die dem orpheischen Traum nie gerecht zu werden vermag: Schriftsteller ohne Literatur zu sein, Literatur ohne Sprache, Denken ohne Struktur.

Der unerfüllbare Traum einer Sprache, die ganz an das Erleben herankommt, es ganz vermittelt und uneingeschränkt auslöst, nimmt seine Gestalt, wie auch Barthes feststellt, bereist früher an. Die Bedingung der modernen Problematisierung der Sprache und Schrift liegt in der Aufwertung des individuellen Maßes wie es von Johann Gottfried Herder beschrieben wird: „Jeder Mensch hat ein eigenes Maß, gleichsam eine eigne Stimmung aller seiner sinnlichen Gefühle zu einander.“ An derselben Stelle auch: „So daß jeder Mensch zuletzt eine Welt wird, zwar eine ähnliche Erscheinung von aussen, im Innern aber ein eignes Wesen, mit jedem andern unausmeßbar. "16 Herder ist nicht der Einzige, der im ausgehenden 18. Jahrhundert die Vorstellung vertieft, dass jeder Einzelne von uns seine eigene originelle Weise hat, Mensch zu sein. Die Überzeugung, dass es eine bestimmte Weise gibt, Mensch zu sein, welche die je eigene Weise ist, findet Unterstützung und setzt neue Maßstäbe sowohl in Bezug auf die Kunst als auch in Bezug auf die Moral. Denn das Freilegen der Idee einer Authentizität geht einher mit der Etablierung der Authentizität als ein Ideal. ${ }^{17}$ Der individuellen Erlebenswelt und dem Unterschied zwischen den Menschen kommt von nun an ein vorher unbekanntes Gewicht zu. ${ }^{18}$

Ebd., S. 10.

15 Ebd., S. 11.

16 Johann G. Herder, „Ideen“, 7. Buch, in: Ders., Sämtliche Werke, hg. v. Bernhard Suphan, Bd. 13, Berlin, 1877-1913, S. 291.

17 Zur Herausbildung der Authentizität als Ideal: Charles Taylor, Quellen des Selbst, Zur Entstehung der neuzeitlichen Identität, übers. v. Joachim Schulte, Frankfurt am Main, 1994, S. 639ff. Ders., Das Unbehagen an der Moderne, übers. v. Joachim Schulte, Frankfurt am Main, 1995, S. 34ff.

18 Wie sehr dieses Empfinden die kommenden Jahrhunderte prägt, zeigt eine Passage in Janet Frames 1963 entstandenem und 2007 posthum veröffentlichtem Roman Dem neuen Sommer entgegen: „Rufen. Und tanzen vor Wut und Schmerz, wenn keine Antwort kommt, wenn wir gewahr werden, dass die 
Die innere Stimme wird zum neuen Maßstab. ${ }^{19}$ Wer sich selbst treu sein will, muss lernen, darauf zu hören, muss fähig sein, sich darauf einzulassen, um dem eigenen Erleben Gültigkeit zu verschaffen und diesem im besten Fall Ausdruck zu verleihen. Die Literatur und Kunst gerät bei diesem Unterfangen, das nicht delegiert werden kann, zur hochqualifizierten Assistentin. Die Schriftsteller vermitteln, indem sie ihrem eigenen Erleben möglichst treffend Ausdruck verleihen, und auch, indem sie das Erleben im Rezipienten selbst wachrufen. Das Innere und die eigne Stimmung werden zu unverzichtbaren Komplizen des bewussten Selbst, die sich aber einer umfassenden Integration in Sprache entziehen.

Die Schreibweise der Moderne entwirft eine vielfältige Architektonik dunkler Innenräume, in denen sich Folgenreiches vollzieht. Es wird nach einer Sprache gesucht, die dem Erleben dieser inneren Geschehen, die der Erfahrung von Leiblichkeit und die dem Unbewussten ${ }^{20}$ angemessen Ausdruck zu verleihen vermag. Dabei stoßen die Sprache und insbesondere die Schriftsprache an Grenzen, welche von den Texten der Moderne inszeniert und wogegen gleichzeitig angeschrieben wird. Dabei kommt den Auslassungspunkten eine bedeutende Funktion zu. Sie werden gerade in Bezug auf die stummen Landschaften ${ }^{21}$ jedes Einzelnen zu Kronzeugen einer verborgenen, aber wirksamen Welt, zu Signalen vielfältiger nicht in Bedeutung übersetzbarer Hohlseiten.

\section{Die Punkte als eine Schreibweise des Seins}

Bisher wurde dargelegt, inwiefern die Transformation der Auslassungspunkte vom Satzzeichen zur Schreibweise und die Herausbildung der Literatur als zentraler Agentin auf der Suche nach einer Sprache des Erlebens historisch und systematisch in Verbindung zueinander stehen. Inwiefern die Punkte in diesem Wandlungsgeschehen zu einer Schreibweise des Seins formieren, dieser Frage möchte ich im Folgenden nachgehen. Wie eingangs erwähnt worden ist, nehmen die Punkte Partei für ein außerhalb des Mitgeteilten Liegendes und zwar in doppeltem Sinn. Zum einen signalisieren die Punktspuren als Schreibweise eine historische Situation und

Einheit, nach der wir messen, einzigartig ist, dass sie keinem anderen ins Herz geprägt ist und niemals geteilt werden kann“ (Janet Frame, Dem neuen Sommer entgegen, übers. v. Karen Nölle, München, 2010).

19 Deutlich wird das auch an Hugo von Hofmannsthal bekanntem, 1902 publiziertem Text „Ein Brief“. Lord Chandos Brief an Francis Bacon artikuliert die Forderungen an eine neue Poetik, die sich aufgrund der Differenz zwischen Erleben und Sprache aufdrängen.

20 Die freigelegten Innenräume des Subjekts, die innere Stimme und eigne Stimmung, werden im 19. Jahrhundert nicht nur von Kunst, von Literatur und Philosophie, sondern insbesondere auch von Medizin und Psychologie neu strukturiert. Auf den Zusammenhang zwischen der Entwicklung der Psychoanalyse und der Verwendung der Auslassungspunkte kann hier nicht weiter eingegangen werden. „Alles, was wir den Anderen zuwerfen, ist in jenem weiten stummen Land gewachsen, von dem wir immerdar umgeben sind“ (Maurice Merleau-Ponty, Das Sichtbare und das Unsichtbare, übers. v. Regula Giuliani, Bernhard Waldenfels, München, 2004, S. 167). 
den vom Textproduzenten in der Schrift vollzogenen „Akt der Solidarität“"22. Dieser Akt der Parteinahme gilt im Falle der hier im Interesse stehenden Punktspuren einer Sprachauffassung, die Sprache als begrenzte begreift und zwischen Erleben, Ausdruck und Verstehen Zäsuren vorstellt. Als Schreibweise des Seins signalisieren die Punkte zugleich Möglichkeit und Unmöglichkeit von Sprache. Sie inszenieren eine Grenze, durch welche der Raum, der diese Grenze umspielt, entworfen bzw. sichtbar gemacht wird. Wo die Punkte als Schreibweise Verwendung finden, stellen sie ein Außen vor, das nicht in Sprache aufgeht. Die Punkte inszenieren dabei Gelingen und Scheitern des Anspruchs, eine Ausdrucksweise zu schaffen, die dem je eigenen Maß gerecht zu werden vermag.

Inwiefern aber steht die Suche nach dem originellen Erleben im Innern des Subjekts in einem Verhältnis mit dem Ganzen? Inwiefern hängt die Schreibweise, welche die Suche nach einer angemessenen Artikulation für die ganz eigenen Weise, Mensch zu sein, zur Schau stellt, mit der Schreibweise zusammen, die für einen unbestimmten Raum, für das Ganze, für eine Realität außerhalb der Sprache Partei nimmt? In Else Lasker-Schülers Gedicht „Weltflucht" findet sich eine Zeile, die diese zunächst seltsam anmutende Relation verdichtet. Dort heißt es: „Ich will ins Grenzenlose zu mir zurück." Die Suche nach dem Grenzenlosen führt nicht hinaus, sondern zurück in die Intimität des Innersten.

Zur Klärung der Frage des Verhältnisses zwischen innerer Stimme und äußerer Realität lassen sich zudem Jean-Jacques Rousseau und Martin Heidegger heranziehen. Die beiden Philosophen fügen sich wie eine Klammer um das Projekt Moderne herum. Rousseaus Text kommt ohne Punktspuren aus. Darin aber lässt sich früh jener Zusammenhang zwischen innerer Stimme und Natur ablesen, dessen ideengeschichtlicher Werdegang sich in der Folge so erfolgreich entwickeln wird. Bei Heidegger allerdings treten die Punkte in exemplarischer Weise auf und führen so den reflektierten Zusammenhang von Erleben, Stimmung und unbestimmtem Raum schriftbildlich vor. Dass Rousseau die Punkte nicht verwendet, erstaunt nicht. Seine Reflexion der Verbindung von innerer Stimme und Natur leistet gedanklich das, wofür in der Folge eine angemessene Ausdrucksweise gesucht wird.

In Träumereien eines einsamen Spaziergängers legt Rousseau die Problematik der Moral so dar, als gehe es darum, einer Stimme der Natur im Inneren des Menschen möglichst zu folgen. Die Stimme wird häufig übertönt durch Ablenkungen wie Leidenschaften und Reize. Die Wiederherstellung der authentischen Verbindung zwischen dem Selbst und dieser verschütteten Stimme verspricht innere Ruhe und gesellschaftliches Wohl. Der enge Kontakt zu sich selbst wird von Rousseau zur Bedingung für Freude und Zufriedenheit und auch für die Realisierung aller anderen Moralvorstellungen erhoben. Die Bezugnahme von uns auf diese unsere je eigene innere Stimme der Natur wird von Rousseau als „sentiment de l'existence“ bezeichnet: „Das Gefühl des Daseins, von jeder anderen Empfindung entblößt, ist an sich selbst ein köstlicher Genuß der Zufriedenheit und Ruhe, der allein zugäng-

22 Barthes, Am Nullpunkt der Literatur, S. 20. 
lich wäre, dieses Dasein dem teuer und angenehm zu machen, der alle sinnlichen und irdischen Eindrücke, die unaufhörlich kommen, uns davon abzulenken und die Süßigkeit desselben hier zu trüben, von sich fernhalten vermöchte. Aber die meisten Menschen, die von Leidenschaften ohne Ende hin und her geworfen werden, kennen diesen Zustand kaum, und da sie ihn nur wenige Augenblicke unvollkommen genossen haben, so haben sie nur eine dunkle und verworrene Vorstellung davon behalten, die ihnen den Reiz desselben nicht fühlbar machen kann. “" ${ }^{23}$ Das Gefühl des Daseins als Gefühl des Verbundenseins mit der Natur verspricht Ruhe und Zufriedenheit. Um sich auf dieses Gefühl einlassen zu können, bedarf es einer Aufmerksamkeit gegen Innen, die einen als Teil eines Ganzen, als Teil der Natur erleben lässt, was von Rousseau als ein köstlicher Genuss geschildert wird.

Die Offenbarung des Sichbefindens inmitten des Seienden im Ganzen verläuft auch in Heideggers Diktion über das persönliche Erleben. Langeweile zum Beispiel oder die Freude an der Gegenwart eines geliebten Menschen ermöglichen uns diese Offenbarung: „Geschieht im Dasein des Menschen solches Gestimmtsein, in dem er vor das Nichts selbst gebracht wird? Dieses Geschehen ist möglich und auch wirklich - wenn gleich selten genug - nur für Augenblicke in der Grundstimmung der Angst. Mit dieser Angst meinen wir nicht die recht häufige Ängstlichkeit, die im Grunde der nur allzu leicht sich einstellenden Furchtsamkeit zugehört. Angst ist grundverschieden von Furcht. " ${ }^{24}$ In Was ist Metaphysik? führt Heidegger am Beispiel der Angst vor, wie einen ein Empfinden, ein Gestimmtsein, vor das Seiende im Ganzen führen kann. Das Erleben ist individuell und doch hält es den Einzelnen, so der Gedanke von Heidegger, in das Ganze hinein. Um das Empfinden, das sich in diesen Augenblicken einstellt und das sich der prädikativen Sprache widersetzt, im Text Ausdruck zu verleihen, greift Heidegger auf die Auslassungspunkte als bereits etablierte Schreibweise zurück.

Die Auslassungspunkte werden in der Beantwortung der Frage „Was ist Metaphysik?" als Operatoren eingesetzt, um die Grenze der ,Sprache der Logik' zu verdeutlichen und gleichzeitig über diese Grenze hinaus den Raum anzuzeigen, vor dem sich Sprache erst kundtut. Im Rückgriff auf das Beispiel der Angst legt Heidegger nahe, dass der Mensch sich jedenfalls für Momente in einem "Gestimmtsein“ wiederfindet, welches nicht auf den Begriff gebracht werden kann. Im Gegensatz zur Furcht, die einen vor etwas Bestimmtem erfasst, ist der Mensch in der Angst ins Nichts hineingehalten und also weiß er nicht, wovor er Angst hat, auch wenn er das Geschehen der Angst kennt. Die bekannte Unbestimmtheit des Empfindens, das Hineingehaltensein in ein spezifisches Gestimmtsein, das dem Erleben vertraut ist, der Sprache der Logik, wie es bei Heidegger heißt, aber unzugänglich ist, wird mit den Punkten ins Schriftbild geholt. So wird jener außer-sprachliche Raum mit Sprachmaterial doch noch umrissen und ein spezifisches Leseerleben vorstruktu-

23 Jean-Jacques Rousseau, „Träumereien eines einsamen Spaziergängers“, Fünfter Spaziergang, in: Ders., Schriften, hg. v. Henning Ritter, Bd. 2, München, 1978, S. 637-760, hier S. $699 \mathrm{ff}$.

24 Martin Heidegger, Was ist Metaphysik?, Frankfurt am Main, 2007, S. 34. 
riert: „Zwar ist die Angst immer Angst vor ..., aber nicht vor diesem oder jenem. Die Angst vor ... ist immer Angst um ..., aber nicht um dieses oder jenes. Die Unbestimmtheit dessen jedoch, wovor und worum wir uns ängstigen, ist kein bloßes Fehlen der Bestimmtheit, sondern die wesenhafte Unmöglichkeit der Bestimmbarkeit.“" ${ }^{25}$ Oder auch: „In der Angst liegt ein Zurückweichen vor ..., das freilich kein Fliehen mehr ist, sondern eine gebannte Ruhe. Dieses Zurück vor ... nimmt seinen Ausgang vom Nichts. Dieses zieht nicht auf sich, sondern ist wesenhaft abweisend. “26

Die Schreibweise des Seins inszeniert einen Raum oder eine Wirklichkeit außerhalb von Sprache und Stil. Sie signalisiert eine Ontologie, worin die Grenze der Sprache nicht auch die Grenze des Erlebens bedeutet. Unter anderem mit Hilfe der Punktspuren wird die Begrenzung, die inszeniert wird, gleichzeitig schriftbildlich doch auch überwunden. Was der begrifflichen Sprache verschlossen ist, findet durch die Visualität der Materialreste des Vor- oder Nicht-Sprachlichen doch noch Eingang in das Textgeschehen. Die Punktspuren brechen in der Schrift den Raum zwischen den Lettern und dem Nichts auf und führen damit den außer-sprachlichen Raum als Bedingung sprachlicher Verständigung vor.

\section{Die Punkte und die Art des Lesens}

Die frühe Regel der Interpunktion ,Schreibe wie du sprichst' wird in jenen Jahrzehnten, in denen historisch einerseits das Interesse am Innern und an den darin waltenden Stimmen und Stimmungen wächst und andererseits, und zum Teil damit verbunden, sich ein neues Sensorium für die Problematik von Sprache entwickelt, für die Verwendung des Auslassungszeichens neu formuliert zu: ,Empfinde, wenn du liest.' Damit rückt das Augenmerk auch noch einmal neu auf die lesende Person. Als Schreibweise zengen die Punkte nicht nur von einer bestimmten Sprachauffassung, sie erzengen ihrerseits eine Lektüre-Erfahrung, die diese Auffassung unmittelbar plausibel machen will.

Damit die Inszenierung erfolgreich ist und sich die Funktion der Schreibweise erfüllt, bedarf es des Einbezugs der Textrezeption. Signale sind zuallererst darauf angelegt, die Aufmerksamkeit eines Lesers, einer Leserin in Bann zu ziehen und aufzuklären, noch bevor der Aussagegehalt eines Textes begriffen worden wäre. Ein Schriftbild voller Punktspuren zum Beispiel visualisiert Lücken im Gefüge und prägt den Vollzug, noch bevor die lesende Person wirklich weiß, worum es im Text inhaltlich geht. Die vielen Dialoge in Texten der Moderne, die unablässig von Punktspuren umspielt werden, wie zum Beispiel im Stück Wer hat Angst vor Virginia Woolf...? von Edward Albee, vermitteln eine Brüchigkeit des Grundes,

$25 \mathrm{Ebd}$

26 Ebd., S. 37. 
noch bevor die Figuren ihre Verletzlichkeit formuliert haben oder die formalen Eigenschaften im Einzelnen analysiert worden wären. In Stimmungen lesen. Über eine verdeckte Wirklichkeit der Literatur akzentuiert Hans Ulrich Gumbrecht die Relevanz dieses Aspektes für eine gelingende Lektüre-Erfahrung bzw. LektüreAnalyse. Er reformuliert damit in jüngster Zeit, was u.a. Susan Sontag bereits 1966 in „Against Interpretation“ fordert und in der bekannten Formulierung kulminiert: „in place of a hermeneutics, we need an erotics of art.“

Was Barthes für die Schreibweise ausführt, formuliert Gumbrecht 2011 für die Art des Lesens. Er fordert ein Lesen, das der (dritten) Dimension von Stimmung und Form spezifische Aufmerksamkeit entgegen bringt: „Doch worauf ich hinaus will, ist die Tatsache, dass solche textuellen Töne, Atmosphären und Stimmungen nie ganz unabhängig von den materiellen Komponenten der Texte sind, vor allem von ihrer Prosodie - und dass die Texte deshalb in sehr ähnlicher Weise auf die ,inneren Gefühle' ihrer Leser wirken, wie es das Wetter oder Musik tut. Hier liegt der Grund, warum ich glaube, dass die Dimension von Stimmung tatsächlich eine neue Perspektive und Möglichkeit innerhalb der ,Ontologie von Literatur' eröffnet. “27 Gumbrechts Forderung einer Ontologie von Literatur betont, was die Punkte als Schreibweise gleich in mehreren Dimensionen betrifft. Die Punkte nehmen Partei für jene formalen und materiellen Eigenschaften, die das Erleben beeinflussen und die nur partiell in den Kategorien der prädikativen Sprache oder des Stils aufgehen. Wenn hier bisher die visuelle Eigenschaft der Punkte im Vordergrund stand, dann bedeutet das nicht, dass die Punkte nicht auch unter dem Aspekt der Prosodie betrachtet werden können und müssen. Die Pluralisierung vom einen Punkt zu drei, vier oder mehr Punkten in moderner Literatur und Philosophie bedeutet deutlich eine auch rhythmisch neu konzipierte Sprachdynamik. Überreizung und Gehetztsein, Ruhelosigkeit und Panik werden nicht nur bei Céline durch Punktspuren insinuiert.

„Stimmungen lesen ' heißt immer auch, dass wir aufmerksam sind auf die textuelle Dimension der Formen, welche uns und unseren Körper als potentielle physische Realität umgeben und so ,innere Gefühle' auslösen können, ohne dass dabei notwendig eine Ebene der Repräsentation eingeschaltet sein muss (dies kann immer, muss aber nie der Fall sein). ${ }^{28}$ Gumbrecht verweist in seiner Ausführung auf verschiedene Beispiele der Weltliteratur, die unter anderem nur deshalb funktionieren, weil sie uns in unserem Erleben tangieren und dies häufig nicht primär aufgrund des Inhalts, sondern durch die Atmosphäre, den Ton, die Form, die Stimmung. Zentral für die von Gumbrecht dargelegte Leseweise ist der Einbezug des Körpers, der beim Lesen weit stärker affiziert wird als das die gängige Literaturanalyse vermuten lässt. In Bezug auf die Untersuchung der Auslassungspunkte und deren Stellung im modernen Textgeschehen macht diese Erweiterung offensichtlich Sinn.

27 Hans Ulrich Gumbrecht, Stimmungen lesen. Über eine verdeckte Wirklichkeit der Literatur, München, 2011, S. 12.

28 Ebd., S. 13. 
Als eine Schreibweise des Seins nehmen sie Partei für eine Ontologie von Literatur und lassen sich auch nur durch den Einbezug einer Dimension von Nicht-Sprachlichkeit adäquat in die Interpretation und Analyse einfügen..$^{29}$ Gesetzte Punkte verändern das Schriftbild grundlegend und prägen damit die Haltung der lesenden Person zu den um die Punkte herum gemachten Aussagen blitzschnell und entscheidend. Jenseits der Bedeutung wird so in Schrift noch Bedeutung generiert.

Im Folgenden werden Beispiele moderner Literatur vorgestellt, worin der Gebrauch der Auslassungspunkte generell oder an einer bestimmten Stelle auffällt und die Punkte einen Raum oder Horizont sichtbar machen, vor dem sich Bedeutung differenziert. Mit der Setzung der Punkte wird in vielfältiger Weise die Grenze der Sprache angezeigt und gleichzeitig beabsichtigt, den Raum des Sprachlichen über die Bedeutung hinaus auszudehnen.

\section{Bedeutung jenseits der Bedeutung I: Singularitäten (Arthur Schnitzler, Thomas Bernhard, Heinrich Mann)}

In den Beispielen von Arthur Schnitzler, Thomas Bernhard und Heinrich Mann bilden die Punktspuren die Brüchigkeit der sozialen Verhältnisse ab. Sie erinnern in der Schrift an jene Abgründe der Normalität und jene Singularitäten des Erlebens, die nicht umfassend in propositionale Sprache überführt werden können. ${ }^{30}$

Schnitzlers Reigen ist voll von drei vier und mehr Punkten. In der handschriftlichen Fassung des Stücks erscheinen die Punkte in ungeordneter Anzahl. ${ }^{31}$ Diese verweisen nicht mehr nur auf weggelassene Satzteile, sondern fokussieren die Eigenschaft der Sprache, nie alles auf den Punkt bringen zu können. Die Punkte werden dabei zu Signalen, die der Wahrnehmung einer tief in die Sprache selbst

29 Den physiognomischen Aspekt von Satzzeichen als Gegenpol zu den Namen, zu Bedeutung und Ausdruck, unterstreicht auch Adorno: „Gleicht nicht das Ausführungszeichen dem drohend gehobenen Zeigefinger? Sind nicht Fragezeichen wie Blinklichter oder ein Augenaufschlag? Doppelpunkte sperren, Karl Kraus zufolge, den Mund auf: weh dem Schriftsteller, der sie nicht nahrhaft füttert. Das Semikolon erinnert optisch an einen herunterhängenden Schnauzbart; stärker noch empfinde ich seinen Wildgeschmack. Dummschlau und selbstzufrieden lecken die Anführungszeichen sich die Lippen“ (Adorno, „Satzzeichen“, S. 106).

30 Anna-Lisa Dieter (München) weist auf die diesbezügliche Punkte-Verwendung in Stendhals Armance (1827) hin. Von Octave, dem Protagonisten, steht nur fest, dass er nicht ist wie die Anderen. Wodurch er sich unterscheidet, das zu erläutern gelingt aber kaum. Weder dem Erzähler noch der Mutter oder anderen Figuren ist es möglich, die „singularités“, eventuell auch das Geheimnis um Impotenz, in propositionale Sprache zu fassen. Die Punkte fungieren doppeldeutig, indem sie das Geheimnis um Octave gleichzeitig sichtbar machen und geheim halten bzw. anzeigen, was nicht offenzulegen, nicht auf den Begriff zu bringen ist. Dazu: Anna-Lisa Dieter: Verwundbare Geschichte: Die Poetik der Restauration bei Stendhal und Balzac. (Publikation in Vorbereitung.)

31 Abdruck der Handschriften von 1896/1897 in: Arthur Schnitzler: Ein Liebesreigen. Die Urfassung des „Reigen“, hg. v. Gabriella Rovagnati, Frankfurt am Main, 2004. 
eingeschriebenen Charakteristik Ausdruck verleihen. Sie stecken im Schriftbild sichtbar einen Raum ab, worin Sprache als eine eingebettete erscheint:

Der Gatte: Woran mein Schatz?

Die junge Frau: An ... an ... an Venedig. ...

Der Gatte: Die erste Nacht ...

Die junge Frau: Ja ... so ...

Der Gatte: Was denn - ? So sags doch!

Die junge Frau: So lieb hast du mich heut!

Der Gatte: Ja, so lieb.

Die junge Frau: Ah ... Wenn du immer ...

Der Gatte in ibren Armen: Wie?

Die junge Frau: Mein Karl!

Der Gatte: Was meinst du? Wenn ich immer ...

Die junge Frau: Nun ja.

Der Gatte: Nun, was wär denn, wenn ich immer...?

Die junge Frau: Dann wüsst ich eben immer, daß du mich lieb hast. ${ }^{32}$

Die Kommunikation zwischen dem Gatten Karl und der jungen Frau gibt inhaltlich nicht viel her. Erst die Auslassungspunkte evozieren beim Lesenden den Eindruck, dass sich hinter der abgebildeten und scheinbar einfachen Kommunikationsstruktur eine Tiefe verbirgt, die in der Hoffnung auf echte Anteilnahme und aus Angst vor Verletzung die Erlebenswelten der beiden Sprechenden komplex und kontrovers bestimmt. Erst aufgrund der Punkte wird die Beziehung zwischen Mann und Frau hier als eine disparate lesbar. Hinter (oder neben oder vor oder unter) den in aussagekräftiges Sprachmaterial eingebundenen Aussagen schwingen weitere Qualitäten mit, so implizieren die Punkte, die sich nicht in gängiges Sprachmaterial fassen lassen.

Die Punktspuren inszenieren hier, wie bereits früher angedeutet, einen Raum um die prädikative Sprache herum. Ihre Verwendung sprengt den Rahmen der bisher traditionellen Schreibweise, indem sie mit Sprachmaterial auf die Materialität von Sprache aufmerksam macht. Wenn Adorno beklagt, dass die Verwendung der Punkte „eine Unendlichkeit der Gedanken und Assoziationen“ suggeriere, „welche der Schmock nicht hat", dann stimmt das hier nur bedingt. Die Punkte sondieren hier für die Möglichkeit der Unendlichkeit von Gedanken und Assoziationen und inszenieren gleichzeitig die Unmöglichkeit dieser Unendlichkeit in der begrifflichen Sprache. Die Punkte deuten zudem an, dass der prädikative Gehalt von Sprache und das Erleben nicht identisch sind, dass zwischen dem Sagen-Wollen und dem Gesagten eine kontinuierliche Zäsur vorliegt. Dabei werden die Punkte

32 Arthur Schnitzler, Reigen, Frankfurt am Main, 1960, S. 57. 
ihrerseits zum Ausdruck einer bestimmten Sprachauffassung, zu einer Schreibweise des Seins.

Eine Spielart dieser Schreibweise zeigt sich auch in Thomas Bernhards Erzählung Jauregg. Auch hier unterlaufen die Punktzeichen den Sinn prädikativer Sprache. Die Punkte artikulieren eine eigene, widerläufige Aussage. Die als Monolog angelegte Erzählung beginnt ohne auffällige Zeichensetzung. Je spürbarer der Text die Not des Protagonisten zum Ausdruck bringt, je brüchiger sich der Grund zeigt, worauf sich die Sprache bewegt, desto gehäufter erscheinen die Punkte und entfalten einen gegenläufigen Subtext. Der Inhalt des Gesagten respektive des in der IchForm Geschriebenen wird durch das Schriftbild mit den eingelassenen Punkten auf seine Fragilität hin überführt. Noch bevor wir den Inhalt entziffern, nehmen wir visuell die Brüchigkeit der Sprachordnung wahr. Die folgende Passage erscheint kurz vor Ende des Textes:

... Aber ich weiss auch, dass es lächerlich ist, eine verzweifelte Existenz zu führen, auch nur die Feststellung zu machen, man führe eine verzweifelte Existenz, ist lächerlich, wie ja der Gebrauch des Wortes „Verzweiflung“ an sich schon lächerlich ist... und wie, wenn man es überlegt, alle Wörter, die man gebraucht, auf einmal lächerlich werden... aber ich erlaube mir keine Abschweifung, lächerlich oder nicht, meine Existenz ist eine verzweifelte, wie es ja in den jaureggschen Steinbrüchen nur verzweifelte Existenzen gibt, nicht eine einzige nicht verzweifelte, aber wie die anderen ist auch die meine, den Umständen in den jaureggschen Steinbrüchen entsprechend, apathisch geworden, anspruchslos... Ich sage mir, ich bin zwar verzweifelt, aber ich muss nicht verzweifeln, grundsätzlich bin ich ja immer verzweifelt, grundlegend, aber ich muss nicht verzweifeln... ${ }^{33}$

Der Abschnitt thematisiert gleich auf mehreren Ebenen die Problematik von Sprache, Mündlichkeit und Erleben. Eine erste Spannung ergibt sich in der zitierten Stelle zwischen dem wiederholten Kreisen um die empfundene Verzweiflung und dem Urteil, dass die Verwendung des Wortes „Verzweiflung“ und überhaupt die Verwendung aller Wörter lächerlich sind. Trotz dieses Urteils fährt das Ich fort, die Verzweiflung zu thematisieren, so als ob die Feststellung, dass das Reden von Verzweiflung unsinnig sei, ohne Nachhaltigkeit ist. Eine weitere Spannung ergibt sich inhaltlich auch zwischen der wiederholten Andeutung, dass das Ich verzweifelt sei, aber nicht verzweifeln müsse. Diese Widersprüchlichkeit erfährt durch die Satzzeichen eine weitere Schärfe. „Ich muss nicht verzweifeln“, heißt es, aber durch die gesetzten Punkte ist neben der Stimme des Protagonisten, der sich an den Ort seiner Kindheit zurück gewagt hat und das Gespräch mit Verwandten und Ansässigen vergebens sucht, noch eine weitere Stimme zu vernehmen, die sich den Rationalisierungsversuchen widersetzt und dem Verzweifeln in dieser Passage wider alle Rationalität immer mehr Raum verschafft. Zuletzt wird die Spaltung zwischen Aussagegehalt, Sprechführung und Zeichensetzung und also zwischen Denken,

33 Thomas Bernhard, "Jauregg“, in: Ders., Prosa, Frankfurt am Main, 1971, S. 49-64, hier S. 59 [Herv. i.O.]. 
Verhalten und Erleben noch weiter durch eine Kursivsetzung im Schriftbild zugespitzt: „Ich muss nicht verzweifeln..." heißt es da und doch ist in der Lektüre, im Vollzug von Text und Schriftbild die Verzweiflung unablässig spürbar.

Deutlich übernehmen die Punktspuren an dieser Stelle eine Katalysatorfunktion, durch welche dem Lesenden eine Differenz zwischen Rationalität und Emotionalität, zwischen Sprechen und Erleben offenbar gemacht wird. Während bereits die prädikative Sprache eine Differenz zwischen Urteil und Verhalten markiert, zeigen die Punktspuren in der Schrift Wirkungen des Erlebens des Protagonisten an, die nicht in Bedeutungen übersetzt werden können. ${ }^{34}$

In Heinrich Manns Roman Der Untertan gibt es keine Stelle, an der die Punkte besonders hervorgehoben sind. Weder treten sie in einer Passage besonders gehäuft auf, noch inszeniert sich an einer einzigen Punktsetzung die Brüchigkeit des Grundes ganz offensichtlich. In Manns Roman nisten sich die Punkte wenig opulent, aber alle zwei, drei Seiten wiederkehrend, wie beiläufig, in die Satzreihen ein. Sie stellen hier nur zurückhaltend, aber dafür unaufhörlich den Abgrund der scheinbaren Normalität dar.

Die Analyse der sich über die ersten hundert Seiten erstreckenden Punktsetzung macht deutlich, dass diese ausschließlich im Zusammenhang mit dem Protagonisten Diederich Hessling verwendet werden. Zwar kommen sie auch in der Rede anderer Figuren vor, aber stets nur in der Rede, die sich an Diederich richtet oder aber in dessen eigener. Auch dort, wo die Punkte in einer Passage erscheinen, die aus der Perspektive des Erzählers verfasst ist, stehen die Punkte stets in einem Bezug zum Protagonisten. Sein Name, Diederich, Diedel oder Hessling fällt stets unmittelbar im Satz vor oder nach dem Punktezeichen.

Die „Geschichte der öffentlichen Seele in Deutschland“, wie ein von Mann zuerst vorgesehener Untertitel des Romans heißt, skizziert an den Punkten durchaus eine ,Seelen'-Dimension, die nicht nur den Einzelnen prägt, sondern darüber hinaus einen Typus, der eine ganze Gesellschaft korrumpiert. Im Aufsatz „Reichstag“ von 1911 wird dieser Typus von Heinrich Mann beschrieben als „dieser widerwärtig interessante Typus des imperialistischen Untertanen, des Chauvinisten ohne Mitverantwortung, des in der Masse verschwindenden Machtanbeters, des Autoritätsgläubigen wider besseres Wissen und politischen Selbstkasteiers“. ${ }^{35}$ In Der Un-

34 Ich danke Helmut Lethen (Wien) für den Hinweis auf die Setzung der Auslassungspunkte in Paul Valérys Text Monsieur Teste (1893). Der Protagonist Teste hält nichts von einer emotionalen Ausdrucksweise. Die „Intensität der Gefühle“ eigne sich nicht für den Austausch zwischen Menschen. Profilieren könne man sich nur durch den Scharfsinn des grauen Intellekts. Als Teste von starken Schmerzen heimgesucht wird, kommentiert er sein Empfinden als „nichts Besonderes“. Sein Mono$\log$ ist schriftbildlich durchbrochen von Punktspuren. Diese zeigen einerseits die Unmöglichkeit an, Schmerz narrativ zu fassen und in Bedeutung zu übersetzen und agieren andererseits im Text doch dafür, dass der Lesende bemerken kann, dass da Schmerz wirkt. Dazu ausführlich: Helmut Lethen, „,Der Schmerz trägt keine Bedeutung' (Paul Valéry) oder: Gibt es Ereignisse, die den Kulturwissenschaften den Atem verschlagen?", Vortrag in Zürich, 20.5.2011, (Publikation in Vorbereitung).

35 Heinrich Mann, „Reichstag“, in: Ders., Macht und Mensch. Essays, Frankfurt am Main, 1989, S. 2631, hier S. 31. 
tertan wird dieser Typus an der Figur des erwähnten Protagonisten festgemacht. Sein Leben als Kind, dann in der Schule, im Militär, in der Geschäftswelt, in Familie und Politik wird über sechs Kapitel hinweg entfaltet. In den ersten vier Kapiteln sind die Punkte ausschließlich auf die Figur Diderichs bezogen. Erst in den letzten Kapiteln werden die Punkte auch in der Rede anderer Figuren sichtbar, allerdings bleibt auch da die Verbindung von gesetzten Punkten und vermittelter Doppelbödigkeit bestehen. Eine Ausnahme bildet die allererste Punktsetzung. Da stellen die Punkte zwar ebenfalls verheimlichte Verhaltensweisen zur Schau, hier aber betrifft es noch jene zwischen den Eltern des erst heranwachsenden Diederichs:

Mit der gefühlsseligen Art seiner Frau war Hessling durchaus nicht einverstanden. Sie verdarb das Kind fürs Leben. Übrigens ertappte er sie geradeso auf Lügen wie den Diedel. Kein Wunder, da sie Romane las! Am Sonnabendabend war nicht immer die Wochenarbeit getan, die ihr aufgegeben war. Sie klatschte, anstatt sich zu rühren mit dem Mädchen ... Und Hessling wußte noch nicht einmal, daß seine Frau auch naschte, gerade wie das Kind..$^{36}$

Die nächste Stelle, an der Punkte erscheinen, handelt von der Begegnung mit Göppel und dessen Tochter Agnes. Diederich fühlt sich wohl und unwohl, er stimmt Göppel in allen Belangen zu, respektiert ihn aber nicht, er will der jungen Frau imponieren und sucht ihre Nähe, gleichzeitig geht er ihr aus dem Weg und behandelt sie herablassend. Wo die Punkte erscheinen, spielt das Verhältnis von Unterordnung und Machtausübung eine Rolle. Im Untertan stecken die Punkte jenes Feld ab, worin sich subtile, zerstörerische Gewalt gegen sich und andere anbahnt:

Diederich nahm innerlich Partei für sie [Agnes]. Er hatte eine Wallung von Heldentum: er hätte machen wollen, daß sie alles dürfte, daß sie glücklich war und es ihm dankte ... Da fragte Göppel ihn, ob er in das Konzert wolle. „Ich weiß es nicht“, sagte er verächtlich und sah Agnes an, die sich vorbeugte. „Was ist das für eins? Ich gehe nur in Konzerte, wo ich Bier trinken kann.“ „Sehr vernünftig“, sagte ein Schwager des Herrn Göppel. Agnes hatte sich zurückgezogen und Diederich bereute seinen Ausspruch. ${ }^{37}$

\section{Bedeutung jenseits der Bedeutung II: Übergänge (Arthur Schnitzler und Giuseppe Tomasi di Lampedusa)}

Die Punkte in den beiden hier im Folgenden vorgestellten Passagen in Texten von Arthur Schnitzler und Giuseppe Tomasi di Lampedusa bespielen die Übergänge zwischen Wachen und Schlafen, zwischen Leben und Tod. Sie orchestrieren eine Kontinuität, die sich durch die vielfältigen Zonen sprachbewusster und stummer Landschaften hält. Wie fühlt sich Schlaf an oder das Sterben? Wie kann die intimste

36 Heinrich Mann, Der Untertan, Berlin, 1946, S. 6/7.

37 Ebd., S. 17/18. 
Empfindung in eine soziale Sprache übertragen werden, möglichst ohne Verlust jener spezifischen Qualität und Stimmung, die dem Erleben eigen ist? Die Punkte zielen auf eine Ausdehnung des noch Zugänglichen. Sie versuchen, die Grenze der noch vermittelbaren Erlebensmomente außer Kraft zu setzen. Wenn die Figuren tatsächlich nicht mehr sprechen und erzählen, dann übernehmen die Punktspuren die Orientierung und nehmen uns mit in den Fiebertraum von Felix, in den Todeskampf von Don Fabrizio.

In der Erzählung Sterben von Arthur Schnitzler kommen die Punkte nur einmal zu gehäuftem Einsatz. Die Krankheit von Felix ist hier bereits fortgeschritten. Mit Marie, die eingeschlafen ist, ist er auf der Rückfahrt nach Wien. Felix will sich klar werden, aber die Gedanken lassen sich nicht scharf fassen:

Felix hatte das Bedürfnis, sich vollkommen klar zu werden; aber seine Gedanken wollten keine scharfen Umrisse gewinnen. Er überlegte. Also hier liegt ein schwerkranker Mensch... Der war jetzt im Gebirge, weil dort die schwerkranken Menschen im Sommer hingehen... Und da ist seine Geliebte, und die hat ihn treu gepflegt, und nun ist sie müde davon... So blaß ist sie, oder macht das nur das Licht?... Ach ja, die Lampe brennt ja schon da oben. Aber draußen ist's noch nicht ganz dunkel... Und nun kommt der Herbst... Der Herbst ist so traurig und still... Heute abend werden wir wieder in unserem Wiener Zimmer sein... Da wird es mir vorkommen, als wäre ich nie weg gewesen... Ach, es ist gut, daß Marie schläft, ich möchte sie jetzt nicht reden hören... Ob wohl auch Leute vom Sängerfest im Zuge sind?... Ich bin nur müde, ich bin gar nicht krank. Es sind viel Kränkere im Zug als ich... Ach, tut die Einsamkeit wohl... Wie ist nur heute der ganze Tag vergangen? War denn das wirklich heute, daß ich in Salzburg auf dem Diwan lag? Das ist so lange her... Ja, Zeit und Raum, was wissen wir davon!... Das Rätsel der Welt, - wenn wir sterben, lösen wir es vielleicht... Und nun klang ihm eine Melodie ins Ohr. Er wußte, daß es nur das Geräusch des fahrenden Zuges war... Und doch war es eine Melodie... Ein Volkslied... ein russisches, eintönig... sehr schön... ${ }^{38}$

Die Punkte dienen hier als Mittel, um den Monolog des Kranken auch dort noch fortzusetzen, wo der Schlaf bereits eingesetzt hat, wo sich die bewusste Sprachmächtigkeit des Subjekts auflöst. Felix sinkt in einen fiebrigen Schlaf und wir lesen, geführt von den Punkten, weiter in sein Unbewusstes hinein und nehmen die Musik stärker wahr als den Perspektivenwechsel, der sich in der Erzählweise vollzieht und von der ersten Person zur dritten Person wechselt. Wir können mit Felix mitfühlen und greifen gleichzeitig auf unsre eigenen Erfahrungen beim Einschlafen zurück. Hinein in den Schlaf, in den Traum, in das Innere.

Der Roman Der Leopard weist erst am Ende die Punktspuren auf. Die letzten zehn Seiten beschreiben den Todeskampf des Don Fabrizio. Es beginnt mit dem Blick in den Spiegel. Don Fabrizio erkennt sein Gesicht nicht mehr als sein eigenes. Eine Art Maske verfremdet es: „Es geschieht allen das gleiche: man stirbt mit einer

38 Arthur Schnitzler, „Sterben“, in: Ders., Erzählungen, Frankfurt am Main, 1992, S. 132-222, hier S. $178 / 179$. 
Maske im Gesicht. ${ }^{\text {"39 }}$ Mit der Einsicht in die Vergänglichkeit, in den nahen eigenen Tod, in die Brüchigkeit der eigenen Körperkraft, schleichen sich ins Schriftbild die drei Punkte. Zuerst vereinzelt, dann zunehmend, rhythmisieren sie die aufsteigende Ahnung des Todes und dann den Sterbevorgang selbst. Sie vermitteln zwischen wachem Bewusstsein, Abgleiten in Erinnerung und Heimsuchung fiebriger Halluzination. Zuerst erscheinen sie in Erinnerung an die Söhne und Enkel. ${ }^{40}$ Auf den letzten zwei Seiten sind sie nicht mehr zu übersehen. ${ }^{41}$ Sie treten in der Erinnerung an früher auf und zwar im Zusammenhang mit den Bildern wie „des müden, atemlosen Zuges“, „diese grausame Sonne“, „diese lügnerischen Gesichter“ und „das Hervorbrechen der stürzenden Wassermassen“. Diese Wassermassen werden noch einmal später erwähnt. Wieder sind sie umgeben von Punktspuren, die den unmittelbaren Tod andeuten, den Übergang von einem Empfinden ins andere:

In dem Schatten, der an ihm hochstieg, versuchte er zu rechnen, wie lange er in Wirklichkeit gelebt hatte. Sein Hirn konnte mit der einfachen Rechnung nicht mehr fertig werden: drei Monate, zwanzig Tage, eine Gesamtsumme von sechs Monaten, sechs mal acht, vierundachtzig $\ldots$ achtundvierzigtausend $\ldots \sqrt{ } 840000$. Er fing sich wieder. „Ich bin dreiundsiebzig Jahre alt, in Bausch und Bogen werde ich davon gelebt haben, wirklich gelebt, eine Gesamtsumme von zwei ... drei höchstens." Und die Schmerzen, die Öde, wie viele Jahre waren das? Unnütz, das mühsam zusammenzuzählen - alles, was übrig bleibt: siebzig Jahre.

Er spürte, dass seine Hand die beiden nicht mehr drückte. Tancredi erhob sich eilig und ging hinaus ... Jetzt brach nicht mehr ein Fluß aus ihm heraus, sondern ein Ozean, stürmisch, voller Schaum und entfesselter Sturzwellen... ${ }^{42}$

Interessanterweise geht der Roman noch einen Abschnitt weiter und zwar ohne Punkte. Die Punkte inszenieren den Übergang. Das Empfinden des bereits Toten, der von einer verschleierten Frauengestalt empfangen wird, kommt ohne die Auslassungspunkte aus. Die literarische Parteinahme für den Versuch, alle sprachlichen Möglichkeiten einzusetzen, um auch noch an die individuellsten Erlebensdimensionen heranzukommen, ihnen in und durch die Schrift Raum und Ausdruck zu verschaffen, wird von einer Bildsprache der Theologie abgelöst. Die Intensität des Ringens um Ausdruck, welche die Schreibweise des Seins hervorbringt, ist domestiziert. Die Setzung der Punkte als Schreibweise wäre hier nicht angemessen.

39 Giuseppe Tomasi di Lampedusa, Der Leopard, übers. v. Charlotte Birnbaum, München, Zürich, 1999, S. 293.

40 Ebd., S. 296.

41 Ebd., S. 301/302.

42 Ebd., S. 302. 


\section{Bedeutung jenseits der Bedeutung III: Stumme Landschaften (Marcel Proust und James Joyce)}

In Prousts Auf der Suche nach der verlorenen Zeit tauchen die Punkte nicht häufig auf. Wo sie erscheinen, nehmen sie offensichtlich Partei für das wiederzugewinnende Brachland im Innern der Menschen. Das dreimal nacheinander gesetzte Punktezeichen markiert den Übergang von Außen nach Innen und nimmt den Lesenden mit hinein in Swanns Welt:

Und heute noch, wenn mir in einer großen Provinzstadt oder in einem Stadtviertel von Paris, das ich weniger kenne, ein Vorübergehender, der mir ,den rechten Weg weist ${ }^{\star}$, in der Ferne als Orientierungspunkt den Turm eines Spitals oder Klosters bezeichnet, der die Spitze seines geistlichen Biretts an der Ecke einer Straße erhebt, in die ich einbiegen soll, so wird, wofern meine Erinnerung auch nur den geringsten an jene teure entschwundene Gestalt gemahnenden Zug an ihm findet, der Fremde, wenn er sich noch einmal umblickt, um sich zu überzeugen, daß ich nicht fehlgegangen bin, mit Staunen bemerken, wie ich in völligem Vergessen des geplanten Spaziergangs, der dringenden Besorgung stundenlang unbeweglich im Bemühen des Erinnerns vor dem Glockenturm stehe, da ich auf dem tiefsten Grund meines Innern wiedereroberte Gebiete spüre, deren Untergrund schon trocken wird und zum Wiederaufbau bereit; sicherlich suche ich dann, viel eifriger als eben noch, da ich ihn um Auskunft bat, meinen Weg, ich biege in eine Straße ein ... aber $\ldots$ in meinem Herzen.$^{43}$

Ohne weiteren Kommentar wird hier inhaltlich die Zeit zurückgedreht und wir Lesende sind mit dem Ich in die Gegenwart und Gefilden vergangener Zeiten versetzt.

Auf der Suche nach einer Sprache, welche die Zusammenhänge, Erlebenshorizonte und Bewusstseinszustände sowohl des Einzelnen als auch aller Menschen und aller Lebensweisen der gesamten Weltgeschichte zum Ausdruck bringen will, befindet sich James Joyce. Der Einsatz der Punkte wird in Ulysses zu einer Möglichkeit, über Grammatik und Inhalt hinaus in eine Sprache des Traumes überzuleiten. Eine Briefnotiz von Joyce problematisiert das Verhältnis von Sprache und Existenz und gibt einen Hinweis auf den Anspruch, der an eine Sprache gestellt wird, die den Zuständen, in denen sich die menschliche Existenz vollzieht, Ausdruck verleiht: „Ein großer Teil jeglicher menschlichen Existenz vollzieht sich in einem Zustand, der nicht durch hellwache Sprache, trockennüchterne Grammatik und vorantreibende Handlung wahrnehmbar gemacht werden kann. “44

Ulysses kann als der Entwurf eines kollektiven Traums vorgestellt werden, in dem verschiedenste Realitäten parallel verlaufen und ineinander spielen. Joyce

43 Marcel Proust, Auf der Suche nach der verlorenen Zeit, In Swanns Welt, übers. v. Eva Rechel-Mertens, Frankfurt am Main, 1997, S. 93.

44 James Joyce, Werke, Bd. 6: Briefe II. 1917-1930, übers. v. Kurt Heinrich Hansen, hg. v. Klaus Reichert, Fritz Senn, Frankfurt am Main, 1970, S. 1087. 
greift dabei auf alle möglichen Ausdrucksweisen, Formen und Techniken zurück, von denen hier nur die Verwendung der Auslassungspunkte interessiert. Diese erscheinen an einer Stelle offensichtlich gehäuft. Anders als bei Proust, wo die Punkte den Weg ins Reich des Inneren anzeigen, dienen die Punkte hier bei Joyce unter anderem als Signale des Erwachens und Auftauchens.

Es handelt sich um die Szene zwischen Stephen und Bloom am Ende des zweiten Teils des Werks. Stephen liegt am Boden, Bloom wacht an seiner Seite. Vorher sind sie aus dem Bordell entflohen, dem Reich des Unbewussten. Wie aus einem Schlund ausgespuckt, befinden sich die beiden Figuren nun plötzlich wieder draußen in der Nacht und auf der Straße. Die Punkte ziehen sich sowohl durch die Rede von Stephen als auch durch jene von Bloom. Traum, Erschöpfung, Erinnern an die Mutter von Stephen und an den verstorbenen Sohn Rudy gehen ineinander über:

Bloom: Eh! Ho! (There is no answer. He bends again.) Mr Dedalus! (There is no answer.) The name if you call. Somnambulist. (He bends again and, hesitating, brings his mouth near the face of the prostrate form.) Stephen! (There is no answer. He calls again.) Stephen!

Stephen: (Groans.) Who? Black panther vampire. (He sighs and stretches himself, then murmurs thickly with prolonged vowels.)

Who ... drive ... Fergus now

And pierce ... wood's woven shade ...?

(He turns on his left side, sighing, doubling himself together.)

Bloom: Poetry. Well educated. Pity. (He bends again and undoes the buttons of Stephen's waistcoat.) To breathe. (He brushes the woodshavings from Stephen's clothes with light hand and fingers.) One pound seven. Not hurt anyhow. (He listens.) What?

Stephen: (Murmurs.)

... shadows ... the woods

... white breast ... dim sea.

(He stretches out his arms, sighs again and curls his body. Bloom, holding the hat and ashplant, stands erect. A dog barks in the distance. Bloom tightens and loosens his grip on the ashplant. He looks down on Stephen's face and form.)

Bloom: (Communes with the night.) Face reminds me of his poor mother. In the shady wood. The deep white breast. Ferguson, I think I caught. A girl. Some girl. Best thing could happen him. (He murmurs.) ... swear that I will always hail, ever conceal, never reveal, any part or parts, art or arts... (He murmurs.) ... in the rough sands of the sea ... a cabletow's length from the shore ... where the tide ebbs ... and flows ...

(Silent, thoughtful, alert he stands on guard, his fingers at his lips in the attitude of secret master. Against the dark wall a figure appears slowly, a fairy boy of eleven, a changeling, kidnapped, dressed in an Eton suit with glass shoes and a little bronze helmet, holding a book in his hand. He reads from right to left inaudibly, smiling, kissing the page.)

Bloom: (Wonderstruck, calls inaudibly.) Rudy!45

45 James Joyce, Ulysses, London, 2000, S. 701/702. 
Nach dieser Stelle ist das Schriftbild wieder völlig beruhigt. Kaum ein Absatz bricht das geschlossen wirkende Textformat am Anfang des dritten Teils auf. Das Gespräch, das Bloom und Stephen in der Kneipe führen, wirkt müde und ausdruckslos. Sie verstehen sich nicht wirklich, aber die Müdigkeit lässt sie dagegen auch nicht aufbegehren.

\section{Bedeutung jenseits der Bedeutung IV: Unmittelbarkeit (Denis Diderot und Louis-Ferdinand Céline)}

Wie in Bezug auf Denis Diderot bereits eingangs angedeutet, ist der komplexe Roman Jacques, le fataliste angereichert mit vielen Punktelementen. Dabei nehmen die Punkte kaum eine konkrete repräsentative Funktion ein. Sie werden auch nicht konsequent gleich verwendet, sondern stellen den Versuch dar, den Fluss der Gedanken, die Sprunghaftigkeit der Dialoge und die Unabgeschlossenheit einzelner Passagen zu verstärken. Sie sind hier sicher auch ein Zeichen für den Willen, eine neue, unmittelbare, lebendige Sprache zu generieren, die über jedes traditionelle sprachliche Korsett hinweg den Leser, die Leserin direkt zu erreichen versucht. Abstand will hier möglichst überwunden werden. Sowohl die politische Distanz zwischen den gesellschaftlichen Schichten als auch jene zwischen Autor, Figuren und Lesern, und noch jene zwischen Schrift und Mündlichkeit soll reduziert werden. Die Punkte in Diderots Text beschleunigen den Rhythmus des Lesens. Sie markieren den Wunsch, die Medialität jeder Vermittlung zu überwinden. Ein Bestreben, das später auch Célines Schreiben grundiert.

Louis-Ferdinand Célines Texte sind geprägt vom Versuch, Unmittelbarkeit und Präsenz zu produzieren. Es soll gelingen, das Schriftliche als Mündlichkeit zu inszenieren, wobei die Auslassungspunkte zum zentralen ästhetischen Mittel avancieren. In Reise ans Ende der Nacht, 1932 veröffentlicht, ist eine Häufung der Punktspuren augenfällig. Sie ziehen sich durch das Schriftbild wie der gestiegene Puls des Lebens, das sich unter Bedingungen einer Ausnahmesituation zu behaupten hat. Sie signalisieren den Versuch, eine Sprache zu finden, die das lebenswirkliche Empfinden der dargestellten Figuren trifft. Nicht die Darstellung der Figur interessiert dabei vorrangig, sondern das Interesse gilt der Frage, wie die Darstellung selbst vergessen gemacht werden kann. Es geht Céline um die Schaffung einer fiktiven Mündlichkeit, die ihre schriftliche Bedingtheit ausblenden lässt. ${ }^{46}$

Folgt man den auto-poetologischen Ausführungen von Céline, dann werden die Punkte als Mittel eingesetzt, um im Medium der Graphie konkrete Mündlichkeit vorzustellen. In einem Brief an Milton Hindus vom 17.10.1947 schreibt Céline:

46 Dazu: Andreas Blank, Literarisierung von Mündlichkeit. Lonis-Ferdinand Céline und Raymond Queneau, Tübingen, 1991. 
C’est la ,fleur des nerfs', la mélodie spontanée, la musique de l'âme que j'essaye de capter du langage parlé et de faire passer en écrit. [...] Je veux refaire artificiellement en écrit un langage parlé. Un langage idéal. ${ }^{47}$

In diesem Streben nach einer emotionalen, idealen, intensiv verdichteten, sprechenden Schrift sind die Punkte für Céline unverzichtbar:

Mes trois points sont indispensables! ... indispensables! ... je le répète: indispensables à mon métro .... ${ }^{48}$

Die Punkte sollen einen mitnehmen auf die Reise ganz in der Art eines Verkehrsmittels. Mit ihnen beginnt im übertragenen Sinne auch die Reise ans Ende der Nacht. Die Punktspuren führen in ein Reich des Dunklen, ins Innere des Empfindens der Getriebenen und in jenes der Lesenden. Jedenfalls werden die Punkte bei Céline mit dem Ziel gesetzt, direkt ins Zentrum des Bewusstseins, ins Zentrum des Nervensystems zu führen. Céline vergleicht die Punkte mit den Schienen einer Metro, die ohne Umwege zum Ziel, ins Rückenmark des Lesers, ins Stammhirn der Leserin, führen: „Le truc du ,métro-tout-nerfs-rails-magiques-à-traverses-trois-points“ est plus important que l'atome! “49 Die Punkte sind das magische Transportmittel, das den Lesenden ohne Umweg erreichen kann, direkt und konfrontativ.

An vielen Stellen des Romans Reise ans Ende der Nacht finden sich Punktspuren, die den Abstand ausblenden, die Gegenwärtigkeit realisieren sollen. Die Punkte signalisieren, dass die Erinnerung des Kampfes und der Flucht keinen Rückblick in eine frühere Zeit offenbart, sondern den Einblick in die seelische Verfassung der am Krieg Beteiligten:

Robinson, habe ich gedacht! - Ich heiße Robinson! ... Léon Robinson! - Jetzt musst dus versuchen, jetzt oder nie, hab ich gedacht! ... Nicht wahr! Also ich an einem kleinen Wald lang, und stell dir bloß vor, da treff ich auf unseren Hauptmann ... Stand an einen Baum gelehnt, böse zugerichtet, der Quälgeist! ... War am Verrecken ... Hielt sich die Hose mit beiden Händen und spuckte ... Blutete am ganzen Leib und verdrehte die Augen... Kein Mensch bei ihm. Der hatte sein Fett weg. ... ,Mama! Mama!'. Jammerte er beim Krepieren und pisste Blut. ${ }^{50}$

Léon Robinsons Rede erinnert einen unerwarteten Angriff auf die eigene Kompanie. Die Rückblende wird durch die Punkte gleichzeitig unterbrochen und vorwärts getragen. Der zeitliche Abstand will vergessen gemacht werden, das Erleben als Erleben im Jetzt inszeniert.

47 Brief an Hindus vom 17.10.1947, zit. nach: Milton Hindus, Louis-Ferdinand Céline, tel que je l'ai vu, Paris, 1969, S. 174.

48 Louis-Ferdinand Céline, Entretiens avec le professeur Y, Paris, 2004, S. $115 \mathrm{ff}$.

49 Ebd.

50 Louis-Ferdinand Céline, Reise ans Ende der Nacht, übers. v. Hinrich Schmidt-Henkel, Hamburg, 2003, S. 57. 
Die bisher untersuchten Textstellen machen plausibel, inwiefern in Texten der Moderne die Punkte als eine Schreibweise des Seins aufgefasst werden können. Das heißt nicht, dass der Anspruch erhoben wird, dass jedes gesetzte Auslassungszeichen in modernen Texten eine Schreibweise bzw. eine Schreibweise des Seins bedeutet. Dem für die Punkte geschärften Blick eröffnet sich jedoch in sehr unterschiedlichen Texten der Literatur und Philosophie der Moderne eine verbindende Geste: der Einbezug stimmungswirksamer Möglichkeiten, um das individuelle Erleben möglichst treffend zu vermitteln, ohne es in Bedeutung zu übersetzen. Dabei schärft sich, beabsichtig oder nicht, das Sensorium für die Begrenzungen dieses Versuchs und die Nichtidentität von Sprache und Erleben. Die Punkte fungieren dabei als sprachliches Material, das seine spezifische Funktion ausübt aufgrund der widersprüchlichen Eigenschaft, gleichzeitig bedeutungsvolles und bedeutungsloses Zeichen zu sein.

\section{Von den Punkten zu den Strichen}

Der häufige Einsatz der Auslassungspunkte in Texten der Moderne ist Ausdruck einer systematischen Neubestimmung dieses Satzzeichens. Die Auslassungspunkte verlieren in diesem Prozess die Funktion eines traditionellen Interpunktionszeichens. Sie artikulieren die Suche nach einer neuen, originären und in gewisser Hinsicht unmöglichen Sprache. Sie zeugen vom Versuch, die Präsenz der Stimme in die Schrift zurück zu holen und dem ganz individuellen Erleben Ausdruck zu verschaffen. Dabei inszenieren sie eine Grenze zwischen Innen und Außen, zwischen Erleben und Sprechen und zwischen mündlichem und schriftlichem Ausdruck.

Als Komplizen des in propositionaler Sprache Unaussprechbaren tragen die Punkte in modernen Texten ihrerseits durchaus eigenständig bei zum prädikativen Geschehen. Sie deuten an, dass es etwas gibt, das in Sprache wirkt, was aber sprachlich nicht vollständig in Begriffe gefasst werden kann. Sie konterkarieren eine stumme Landschaft, welche die Sprache bedingt, ohne je exakt definiert oder nachgezeichnet werden zu können. Die Auslassungspunkte zeugen vom Versuch der Überwindung der Differenz und beharren eben so in den Texten auf einer Differenz zwischen Signifikat und Signifikant, indem sie diese Differenz schriftbildlich inszenieren. In dieser Setzung entfalten die Punktspuren eine Schreibweise des Seins, eine Schreibweise der Metaphysik.

Als Schreibweise der Metaphysik müssten die Punkte in der Postmoderne konsequenterweise einer radikalen Kritik unterzogen werden. Als Signal für die Bejahung einer ontologischen Sprachauffassung wird an und mit den drei Punkten schließlich überdeutlich zur Schau gestellt, wogegen die Dekonstruktion andenkt! Als Zeichen der Parteinahme für eine Differenz zwischen Sprache und Erleben ist seine Verwendung in postmodernen, metaphysikkritischen Texten zumindest als problematisch einzustufen. 
„Das geschichtliche Wesen der Satzzeichen kommt daran zutage, daß an ihnen genau das veraltet, was einmal modern war. ${ }^{\text {51 }}$ Adorno skizziert beispielhaft die Verfallsgeschichte des Ausrufezeichens, welches einmal als „Schriftgebärde“ ${ }^{52}$ die graphische Gestalt des Expressionismus prägte, sich aber bald verausgabte. Setzt die Verfallsgeschichte der Punktspuren als einer Schreibweise des Seins parallel zum sich durchsetzenden linguistic turn ${ }^{53}$ ein? Für die neu gedachte Differenz, die différance, müsste, so die naheliegende Annahme, nun auch eine andere Form gefunden werden. Im Folgenden interessiert die Frage, welche Rolle den Auslassungspunkten in postmodernen Texten, insbesondere in Texten der Dekonstruktion von Jacques Derrida zukommt und welche Schlüsse sich daraus noch einmal in Bezug auf die Punkte in der Moderne und Postmoderne ergeben bzw. für eine Ontologie der Literatur.

In einer Notiz von Ludwig Hohl wird der Übergang von den Punkten als einer Schreibweise des Seins zu einer neuen postmodernen Sprachauffassung sehr genau reflektiert und gleichzeitig inszeniert:

Der Mann, der Mensch möchte durch ... hindurch - ja wohin? Durch das Äußere hindurch zum Inneren und Innersten ... Aber in Wirklichkeit bewegt er sich nur, durch das „Äußere“, und kann sich endlich nur selbst vermindern; und es bleibt ihm nur übrig, dasselbe zu wiederholen. ${ }^{54}$

Der Mann, der Mensch möchte durch das Zeichen hindurch ins Innerste, in die Zeichenlosigkeit, (wir erinnern die Zeile aus Else Lasker-Schülers „Weltflucht“), und kommt nur immer wieder im Reich der Zeichen an. Hohl bedient sich nicht nur der drei Punkte, sondern auch noch des Gedankenstrichs und stellt schließlich alles in Frage: Fort! Hindurch, weg - aber wohin? Die Bewegung geht im zweiten Teil der Notiz nicht mehr weiter in einen außer-sprachlich situierten Raum. Die Bewegung verbleibt im Äußeren, in jener Dimension, die nun ihre Eigenschaft, Schichtung zu sein, verliert. Das Innere wird hier als Illusion vorgestellt, welcher sich das denkende Bewusstsein entledigen kann, immer und immer wieder. Hohl verwendet die Punkte, wo die angestrebte und nun mehr traditionelle Bewegung von Außen nach Innen angezeigt werden soll, um dann mit Anführungszeichen jenen unausweichlichen Ort hervorzuheben: das Äußere, die Schrift.

Die Notiz von Hohl bildet den systematischen Übergang von der Ontologie zur Metaphysikkritik der Dekonstruktion exakt ab und nimmt die Neubeurteilung der Punkte vorweg. An die Stelle der Punkte rücken die Anführungszeichen. In Derridas „Sporen“ ist diesbezüglich von der epochalen Herrschaft der Anfüh-

51 Adorno, „Satzzeichen“, S. 107/108.

52 Ebd.

53 Richard Rorty, The Linguistic Turn. Essays in Philosophical Method, Chicago, London, 1992.

54 Ludwig Hohl, Notizen oder Von der unvoreiligen Versöbnung, Notiz 146, Frankfurt am Main, 1981, S. 790 . 
rungszeichen die Rede, die für alle Begriffe aufgerichtet ist. ${ }^{55}$ Die Anführungszeichen sind es zudem auch, die Derrida explizit als Darstellungsmittel nutzt, um den jeweiligen Sinn unabschließbar durch den in der Schwebe gehaltenen Bezug offen zu halten. ${ }^{56}$

Das Zeichen der drei Punkte wird durch das Zeichen der doppelten Anführungsstriche abgelöst. Die Kategorien von Raum und Körperlichkeit werden durch jene von Zeit und Nachträglichkeit ersetzt. Was in der Notiz von Hohl mit Hilfe verschiedener Satzzeichen, insbesondere an den Auslassungspunkten bzw. am doppelten Anführungszeichen, eingefasst wird, liest sich bei Derrida zugespitzt folgendermaßen: „Man muß das Ursprünglich-Sein von der Spur aus denken, nicht umgekehrt. ${ }^{\text {57 }}$ Derrida stellt das Verhältnis von Sein und Sprache, von Innen und Außen nicht auf den Kopf, er hebelt es aus. Es gibt kein Innen und kein Außen. Die Vorstellung von Schichten und Genealogien, die durch Analyse von Verschüttungen freigelegt werden können, wird systematisch kritisiert und durch ein anspruchsvolles Denken ersetzt, das die Abwesenheit vor allem zeitlich denkt, nicht räumlich. Falls in diesem Prozess ein einzelnes Zeichen aufgewertet wird, dann das doppelte Anführungszeichen. Allerdings widerspricht eine Hierarchisierung von Zeichen der Verflechtung als originärer, wie sie von Derrida vorgeschlagen wird: "Genauso wie der Ausdruck nicht kommt und sich als eine ,Schicht' der Gegenwärtigkeit eines vor-ausdrücklichen Sinns hinzufügt, genauso auch kommt nicht das Draußen der Anzeige und affiziert akzidentiell das Drinnen des Ausdrucks. Ihre Verflechtung* ist originär, sie ist keine kontingente Verknüpfung, die eine methodische Aufmerksamkeit und eine geduldige Reduktion auflösen könnten. Bei all ihrer Notwendigkeit stößt die Analyse darin auf eine absolute Grenze." ${ }^{\text {"58 }}$ In dem Augenblick, in dem man „die Möglichkeit eines solchen transzendentalen Signifikats in Frage stellt und wo man erkennt, daß jedes Signifikat auch die Rolle eines Signifikanten spielt, wird die Trennung von Signifikat und Signifikant - das Zeichen - von der Wurzel her problematisch. " ${ }^{\text {59 }}$

Die Bejahung der Welt als Zeichen, das heißt die Bejahung der Zeichenfunktion vor der Ontologie, überführt das binäre Verhältnis von Signifikat und Signifikant in ein Gewebe aus Verweisen. „Diese Verkettung, dieses Gewebe ist der Text, welcher nur aus der Transformation eines anderen Textes hervorgeht. “60 Derridas Denken setzt die „Iterabilität“ umfassend ins Recht. Sinn ist nicht präsent, sondern nur im Durchgang durch alle anderen Elemente des Systems gegeben. Derrida ver-

55 Jacques Derrida, „Sporen. Die Stile Nietzsches“, in: Werner Hamacher (Hg.), Nietzsche aus Frankreich, Frankfurt am Main, Berlin, Wien, 1986, S. 129-168.

56 Gerhard Pasternack, „Repräsentation und Interpretation“, in: Hans Jörg Sandkühler (Hg.): Welten in Zeichen - Sprache, Perspektivität, Interpretation, Reihe Philosophie und Geschichte der Wissenschaften, Bd. 52, Bern, Berlin, New York, 2002, S. 169-192, hier S. 186.

57 Jacques Derrida, Die Stimme und das Phänomen, Frankfurt am Main, 2003, S. 115.

58 Ebd., S. 117 [Herv. i.O.].

59 Jacques Derrida, Positionen, Gespräche mit Henri Rose, Julia Kristeva u.a., Graz, Wien, 1986, S. $56 / 57$.

60 Ebd., S. 67 
neint, dass zu irgendeinem Zeitpunkt, in irgendeinem Sinn ein einfaches Element als solches präsent wäre und nur auf sich selbst verwiese: „Kein Element kann je die Funktion eines Zeichens haben, ohne auf ein anderes Element, das selbst nicht einfach präsent ist, zu verweisen. “61

\section{Die Punkte in der Postmoderne}

Die Punkte erscheinen im Kontext der sprachphilosophischen Überzeugungen, die sich in der zweiten Hälfte des 20. Jahrhunderts durchsetzen, äußerst ungeeignet. Tatsächlich verlieren die Punkte, wie bereits erwähnt, in postmodernen Texten jene Stellung, welche ihnen früher zukommt. Warum aber tauchen sie im Schriftbild der Texte der Postmoderne, insbesondere in Texten von Derrida, überhaupt noch auf? Welche Funktion nehmen sie da ein, wo sie in der Schrift auszumachen sind?

Prominent erscheint der Name des Zeichens als Überschrift eines Bandes, worin Interviews und Aufsätze mit und von Derrida versammelt sind. Points de suspension. Entretiens lautet der Titel der französischen Erstausgabe, - was nach den hier vorgestellten Überlegungen zum Auslassungszeichen in der Moderne zunächst eher überrascht. ${ }^{62}$ Dass die Wahl des Titels nicht auf Derrida selbst zurückgeht, sondern auf die Herausgeber des Bandes, bestätigt den Eindruck, der die weitere Analyse in Bezug auf die Verwendung der Punkte in Derridas Texten ergibt: Die Auslassungspunkte werden durchwegs sehr präzise gesetzt und zwar stets als Ausgangspunkt für eine Dekonstruktion, nicht aber als Ausdruck innerhalb der dekonstruktiven Denkbewegung. Auch wenn Derrida mit der Wahl des Titels der Herausgabe einverstanden gewesen sein wird, fällt auf, dass kein publizierter Text im erwähnten Band den Namen des Zeichens (oder das Zeichen selbst) in der Überschrift trägt. ${ }^{63}$ Auch sonst existiert meines Wissens kein Text von Derrida, der den Namen des Auslassungszeichens im Titel führt. (Dem Anführungszeichen kommt ebenfalls keine solche Stellung zu.) Die Autorität des Namens eines Zeichens ungebrochen aufzurichten, widerspricht dem Anliegen der Dekonstruktion. Diese nimmt genau dort ihren Anfang, wo eine solche Autorität wirkt. ${ }^{64}$ Wo „die unzerstörbare Ein-

61 Ebd., S. 66.

62 Die deutsche Publikation: Jacques Derrida, Auslassungspunkte. Gespräche, übers. v. Karin Schreiner, Dirk Weissmann, hg. v. Peter Engelmann, Wien, 1998.

63 Innerhalb einiger Interviews taucht das Punktezeichen in seiner traditionellen Funktion als Satzzeichen auf, das Abkürzungen markiert.

64 Diesem Anliegen wird im Umschlagstext zur deutschsprachigen Ausgabe keine Rechnung getragen. Dort heißt es: „Durch eine Mannigfaltigkeit des Stils und des Tons - spielerisch, strategisch, leidenschaftlich, analytisch, kämpferisch - verleiht Derrida dem Gespräch die schwebende Offenheit und Unabgeschlossenheit der Auslassungspunkte: die im voraus bedachten Improvisationen werden von Zeiten der Stille interpunktiert, die sie in Atem, in Bewegung halten. Durch Auslassungspunkte werden Grenzen, Normen, Erwartungen überschritten; ihre Flüchtigkeit hält die Gesprächspartner, aber auch die Leser in einer Schwebe, die Raum für eine Antwort des Anderen läßt, für das Versprechen und die Verantwortung des gegebenen Wortes." Diese Formulierungen erscheinen nach der hier vor- 
heit des Wortes privilegiert wird oder aber auch die Autorität des Namens eines Zeichens ungebrochen wirkt, ist eine Vielzahl von Axiomen gegeben, mit deren Überprüfung eine ,Dekonstruktion' den Anfang machen muss (was ich seit dem ersten Teil von De la Grammatologie zu leisten versucht habe) “.65

Die Analyse der Verwendung der Punkte in Derridas Texten macht deutlich, wie genau diese Setzung erfolgt. Konsequenterweise erscheinen die Punktspuren, denen noch die Geste einer Schreibweise anhaftet, in Derridas Texten insbesondere in Fragesätzen. Sowohl in Überschriften als auch innerhalb der Reflexionen und Ausführungen werden die Punkte für jene Fragen verwendet, die wie einen Auftakt bilden zu einer sich daran anschließenden, eingehenden Befragung. Die Punkte wirken wie Hinführungen. In den daran folgenden Schreibbewegungen fehlen sie. In der Grammatologie werden die beiden grundlegenden Fragen der Philosophie mit Auslassungspunkten inszeniert. Die eingeleitete Renovation dieser Fragen kommt dann ohne diese aus.

Im Abschnitt „Das geschriebene Sein“ wird die Frage nach dem Zeichen aufgeworfen und schließlich am Ende des Abschnitts mit den Punkten noch einmal so inszeniert, dass daran der Übergang von den Schreibweisen der Metaphysik zur Dekonstruktion deutlich wird: „Das formale Wesen des Siginifikats ist die Präsenz, und das Privileg seiner Nähe zum Logos als phone ist das Privileg der Präsenz. Diese Antwort ist nicht zu umgehen, sobald die Frage, Was ist das Zeichen?' gestellt wird, das heißt, sobald man das Zeichen auf das Wesen, das ,ti esti hin befragt. Das ,formale Wesen' des Zeichens kann nur von der Präsenz aus bestimmt werden. Man kommt um diese Frage nicht herum, es sei denn, man verwerfe die Form der Frage selbst und nehme den Gedanken auf, daß das Zeichen dieses schlecht benannte Ding ist, das als einziges sich der Grundfrage der Philosophie: ,Was ist ...? entzieht." ${ }^{\text {"66 }} \mathrm{Ab}-$ gesehen davon, dass die Frage zudem auch von Anführungszeichen begleitet wird, mahnen schon die Punkte an eine verblasste Geste, die sich noch zeigt als Bedingung für die angestrebte Neuverortung. Die mäandrierende Schreibweise, die Derrida einsetzt, schreibt die Punkte und die räumlich abgesteckten Leerräume weg. Abwesenheit wird neu gedacht und sucht nach Formen und Ausdrucksweisen, die dieser Veränderung gerecht wird.

Knapp hundert Seiten später wiederholt sich dieselbe Situation im Schriftbild in der Grammatologie: „Wo und wann beginnt ...? Ursprungsfrage. Daß es aber keinen Ursprung, das heißt keinen einfachen Ursprung gibt, daß die Ursprungsfragen

gelegten Untersuchung inadäquat. Dass sie nicht die Dekonstruktion widerspiegeln, sondern die Vorstellungen der Moderne und der Phänomenologie wird überdeutlich, wenn man bei Husserl (nicht bei Derrida!) die Punkte gerade ebenso verwendet sieht, wie sie hier beschrieben werden: „In Beziehung auf jede Thesis können wir und in voller Freiheit diese eigentümliche epoché üben, eine gerwisse Urteilsenthaltung ... Die Thesis wird, außer Aktion' gesetzt, eingeklammert, sie verwandelt sich in die Modifikation ,eingeklammerter Thesis', das Urteil schlechthin in das, eingeklammerte Urteil "“ (Edmund Husserl, Ideen zu einer reinen Phänomenologie und phänomenologischen Philosophie, $\$ 32$ : Die transzendentalphänomenologische epoché, Den Haag, 1950).

65 Jacques Derrida, Wie nicht sprechen. Verneinungen, übers. v. Hans-Dieter Gondek, Wien, 1989, S. 21.

66 Jacques Derrida, Grammatologie, übers. v. Hans-Jörg Rheinberger, Hanns Zischler, Frankfurt am Main, 1974, S. 35/36 [Herv. i.O.]. 
mit einer Metaphysik der Präsenz beladen sind, das dürften wir zweifellos erfahren, wenn wir über die Spur nachdenken. ${ }^{{ }^{67} 7}$ Es gibt keinen einfachen Ursprung. Es gibt keinen Anfang und kein Ende. Es gibt kein Dahinter oder Davor. Derrida insistiert auf der alleinigen differentiellen Konstitution des sprachlichen Zeichensystems. Er tut dies ausgehend von einer Frage, die in Punktzeichen verläuft und im Fragezeichen innehält, bevor dann die neue Schreibweise in Gang kommt und keine Punktspuren mehr auftauchen.

Bemerkenswert ist, dass die Punkte als Schreibweise nicht nur in Fragen auftauchen, sondern auch in Überschriften. ${ }^{68}$ Der Text Wie nicht sprechen. Verneinungen trägt die Punkte zunächst nicht im Titel. In der Ausführung wird auf den Titel, speziell auf die Umstände der Titelsetzung aber eingegangen. Dabei kommen die Punkte ins Schriftbild. Sie bilden auch hier den ersten Anlauf, den Impuls, um der Frage, Wie nicht sprechen' nachzugehen: „Ich wußte also: ich werde es nicht vermeiden können, davon zu sprechen. Doch wie und unter welchem Titel werde ich es tun? Eines Tages erhielt ich eine telefonische Mitteilung in Yale: ich müßte ganz dringend einen Titel geben. Ich habe binnen zwei Minuten improvisieren müssen, was ich zunächst in meiner Sprache getan habe:, Comment ne pas dire ...?' Der Gebrauch des Wortes dire gestattet einen gewissen Aufschub. ,Comment ne pas dire?" kann heißen (dire): wie schweigen, wie nicht sprechen - überhaupt, wie nichts sagen (how to avoid speaking)? ${ }^{\text {(69 }}$ Nur einen Moment lang scheinen die Punkte in der Lektüre auf. Aber schon in der Wiederholung der Ausgangsfrage fehlen sie. Die Umschreibung ist im Gang. Die Punkte sind noch das Resultat einer Improvisation. Sie erscheinen wie eine Reminiszenz.

Die Punkte nehmen in Derridas Texten eine Stellung ein, die der Funktion, die diese als Schreibweise der Moderne haben, angemessen Rechnung trägt. Eine Ausnahme bilden vielleicht jene Texte von Derrida, die ausführlich auf James Joyce Bezug nehmen. Insbesondere in den Überlegungen zum "speechwriting“ bei Joyce und dessen Ausarbeitung des Verhältnisses von Gesprochenem und Geschriebenem, von Stimme und Schrift, tauchen die Punkte bei Derrida gehäuft und in eigenwilliger Setzung auf. „Ulysses Gramophone“ und „Two words for Joyce“ sind angereichert mit Punktspuren, die eher affirmativ als kritisch wirken, so als ob das Schriftbild eine Zuwendung abbildet gegenüber einer Schriftlichkeit, die auch Fremdheit und Befremden auslöst. In diesen Texten jedenfalls unterstreichen die Punkte eine Ambivalenz zwischen Respekt und Bewunderung auf der einen Seite und Unverständnis und Fremdheit auf der anderen, die in "Two words“ gegenüber Joyce ausformuliert wird. ${ }^{70}$ „I'm not sure I like Joyce ... I'm not sure he is liked ...

67 Ebd., S. 130 [Herv. i.O.].

68 Ein Beispiel dafür ist Derridas Text „Wie Meeresrauschen auf dem Grund einer Muschel...“. Inwiefern darin Derrida für die Punkte bzw. die Stimmung Partei nimmt, statt für die Skepsis siehe: Gumbrecht, Stimmungen lesen, S. 173/174.

69 Jacques Derrida, Wie nicht sprechen, S. 33 [Herv. i.O.].

70 Dazu die Studie von Alan Roughley, Reading Derrida, Reading Joyce, Chapter 6, Gainesville, 1999. „Each time Derrida has spoken on Joyce, Joyce's writings operated within what Derrida had to say 
except when he laughs ... he's always laughing ... everything is played out in the difference between several tonalities of laughter." ${ }^{\text {"1 } 11}$

Demgegenüber kommt die schriftliche Auseinandersetzung mit der Literatur von Franz Kafka ohne jede Punktspur aus. In Préjugés. Vor dem Gesetz taucht das Auslassungszeichen zwar auf, allerdings ausschließlich in Zitaten anderer (z.B. bei Lyotard oder bei Husserl) oder als Satzzeichen, das seiner traditionellen Funktion entsprechend Auslassungen im Sinne von Verkürzungen repräsentiert. Die Schreibweise von Derrida trägt die Punkte ebenso wenig vor wie jene von Kafka. Die Problematik der Sprache kann nicht an einem Zeichen deutlich werden, nicht an einem Begriff. Sie erweist sich der lesenden Person über viele Zeilen hinweg, wenn in immer wieder anderer, nur leicht modifizierter Weise die Gleichzeitigkeit, Heterogenität und Widersprüchlichkeit von Bedeutung vorgeführt wird. Die Bewegung des Einschreibens, die Veränderung in der Wiederholung, vollzieht sich nicht punktuell in einem Zeichen, sondern entlang der Zeilen, linear.

\section{Neue Formen, vergleichbarer Traum?}

Die Postmoderne denkt die Abwesenheit anders als die Moderne und sucht für die Darstellung dieses neuen sprachphilosophischen Rahmens nach anderen Schreibweisen. Der Traum, Sprache ohne Sprache zu sein, den Barthes als einen der Moderne ausweist, ${ }^{72}$ ist in der Postmoderne meines Erachtens damit aber nicht vollständig ausgeträumt. Die Divergenz zwischen dem Versuch, das Empfinden des Ereignisses abzubilden und dem Versuch, auf die Möglichkeit des Ereignisses vorzubereiten, ist kleiner als vielleicht angenommen. Im Text Wie nicht sprechen. Verneinungen von Derrida wird dieser Traum als grundlegende Problematik von Sprache vorgestellt. Im Zentrum des Textes steht die Absetzung der Dekonstruktion von der negativen Theologie. Die Bewegung der Absetzung wird darin aber gleichzeitig zu einem Ausloten von gemeinsamen Anliegen. Der Traum wirkt nach. Er wird durch die konsequente Analyse von Derrida nicht nur verworfen, sondern auch bedient. „Nicht, daß alles sich bekundet. Nur die Nicht-Bekundung ist niemals sichergestellt.“73 Die Nichtbekundung ist niemals sichergestellt, denn: Wie nicht sprechen?

Wie sehr der Traum in den Fragen nachwirkt, zeigt zum Beispiel auch eine Antinomie, an welcher sich die Bewegung der Dekonstruktion entfaltet. „1. Man spricht

about those writings, even when Derrida's own spoken words were read from a written text. These textual specters of Joyce's words have important implications for Derrida's investigations of the relationships between speech and writing and his deconstructive overturning of the classical hierarchy privileging speech over writing" (ebd., S. 58).

71 Jacques Derrida, „Two words for Joyce“, in: Derek Attridge, Daniel Ferrer (Hg.), Post-structuralist Joyce. Essays from the french, übers. v. George Bennington, Cambridge, 1984, S. 145-159, hier S. 157.

72 Barthes, Am Nullpunkt der Literatur, S. 12.

73 Derrida, Wie nicht sprechen, S. 38. 
immer nur eine einzige Sprache - oder vielmehr ein einziges Idiom. 2. Man spricht niemals eine einzige Sprache - oder vielmehr, es gibt kein reines Idiom. " ${ }^{74}$ Die Paradoxie zwischen den beiden Aussagen über Sprache evozieren beim Lesenden nicht nur Einsicht in die Verflechtung, sondern sie differenzieren auch das Empfinden dafür. Die Perspektive des stimmungs-orientierten Lesens, das Gumbrecht vorschlägt, ${ }^{75}$ ist, so denke ich, unbedingt auch auf die Texte der Dekonstruktion anzuwenden. Die Differenziertheit, mit der die Texte von Derrida durchkomponiert sind, auch bis ins Schriftbild hinein, lässt einen Einbezug eines solchen Zugangs in der Analyse als unumgänglich erscheinen. Die heftige Ablehnung und die treue Zuneigung, die Verächter und Verteidiger des Anliegens der Dekonstruktion trennt, hat darin vermutlich eine ihrer Ursachen.

Ein Professor für Philosophie meinte einmal, er bekäme stets unverzüglich heftige Kopfschmerzen, es werde ihm richtiggehend schlecht, wenn er Derrida lesen müsse. Was für eine körperliche Reaktion! Die Ausdrucksform in Texten Derridas ist so differenziert durchgeführt und verweigert sich so konsequent dem schnellen Zugang eines auf den Gehalt analytischer Philosophie eingeübten Blicks, dass sie sich nur jener Lesart anvertraut, die sich sorgfältig auf die Stimmung der epoché, auf den Rhythmus der Kreise, die Form von Verzögerung und Verschiebung einlässt. Noch einmal eine Äußerung - dieses Mal jene einer Studentin - die dieses Verhältnis von Stimmung und Inhalt pointiert trifft: Man versteht die Texte von Derrida nur, wenn man sie mag. Gumbrecht beschreibt die von der Dekonstruktion verbreitete Stimmung, die man demnach mögen müsste, als „asketisches Selbstmitleid“76. Diese Beschreibung hätte Derrida vielleicht gar nicht missfallen. Die Einsicht in die Komplizenschaft setzt eine äußerst konzentrierte Reflexion voraus und involviert einen noch in Verhältnisse, die man ganz abzulehnen scheint. Der ironisch-ablehnende Ton Gumbrechts gegenüber der Dekonstruktion ließe sich meines Erachtens gerade mit einer Ontologie der Literatur kritisieren.

Ich erinnere mich an das Aufbrechen jeder einzelnen Seite des Buches Demeure. Maurice Blanchot ${ }^{77}$ von Derrida. Die Verzögerung wurde so zur Erfahrung. Oder denken wir an das Plakat, auf dem der Text Cosmopolites des tous les pays, encore un effort! ${ }^{178}$ publiziert worden ist. Darauf erfährt die graphische Darstellung eine Aufwertung, die den Blick fesselt, die aber die Lektüre des Textes als eines Textganzen und das Verstehen des Anliegens erschwert. Gerade die Verteidiger der Dekonstruktion haben das Anliegen, die Sprache der Philosophie auf ihre Form hin zu reflektieren und diese Aspekte einzubeziehen, sie im Schriftbild umzusetzen, begrüßt und es weitergeführt und umgesetzt. Die Texte von Derrida sind voll von diversen Satzzeichen. Sie sind umrahmt von Motti und Epilogen, von Titeln und Untertiteln und Fußnoten, von Vor-Sätzen und Nach-Sätzen, die alle Teil sind

74 Jacques Derrida, Einsprachigkeit, übers. v. Michael Wetzel, München, 2003, S. 21.

75 Gumbrecht, Stimmungen lesen.

76 Ebd., S. 168.

77 Jacques Derrida, Demeure. Maurice Blanchot, Paris, 1998.

78 Jacques Derrida, Cosmopolites des tous les pays, encore un effort!, Paris, 1997. 
des Gewebes aus Zeichen, Lettern und Lücken, aus Geste, Form, Signatur und Kontext. Die Texte von Derrida sind strukturiert durch Leerzeilen, Einzüge, Absätze, Randbemerkungen. Das Schriftbild ist angereichert mit Kursiv-Setzungen, mit Sprachwechseln, Verweisen und Zitaten. Gerade auch daran wird die Kompositionskunst, die Sprache als Schrift ermöglicht, sichtbar.

\section{Ontologie von Literatur}

Die Ontologie der Literatur ist anderen ontologischen Positionen nicht vorschnell gleichzusetzen. Eine Ontologie von Literatur, so wie sie hier verstanden wird und für die Untersuchung der Funktion der Auslassungspunkte relevant ist, nimmt Partei für einen Sprachbegriff, der die formalen und materiellen, die optischen und prosodischen Aspekte von Sprache in die Analyse von Texten einbezieht. Eine Ontologie von Literatur in diesem Sinne verlangt die Wiedergewinnung der Sprache als einer sinnlichen. Ihr Anliegen ist die Erweiterung des Sprachbegriffs. Die Ontologie der Literatur plädiert für die Suche nach immer neuen Ausdrucksweisen für das intime Erleben des Einzelnen und für die Relevanz dieser Artikulation. Die Punkte als eine Schreibweise des Seins unterstreichen diesen Anspruch. ${ }^{79}$ Sie nehmen Partei für einen nichtsprachlichen Raum, ohne zu behaupten, weder diesen Raum noch die Grenze zu diesem Raum exakt oder allgemein fassen zu können. Sie signalisieren, dass die Grenze der Sprache nicht identisch ist mit dem Erleben. Das macht die Punkte, auch noch wo sie in postmodernen Texten auftreten, zu einem Impuls, an dem der Traum, Sprache ohne Sprache, aufscheint. Es ist der Traum, vor dem der Versuch einer Neuschreibung Sinn macht, vor dem die Analyse auch des unscheinbarsten Zeichens Relevanz erhält.

\section{Literatur}

Abbt, Christine, „Die Auslassungspunkte. Spuren subversiven Denkens“, in: Dies., Tim Kammasch (Hg.), Punkt, Punkt, Komma, Strich? Geste, Gestalt und Bedeutung philosophischer Zeichensetzung, Bielefeld, 2009, S. 101-116.

Adorno, Theodor W., „Satzzeichen“, in: Ders., Noten zur Literatur I, Frankfurt am Main, 2003, S. 106-113.

Barthes, Roland, Am Nullpunkt der Literatur, übers. v. Helmut Scheffel, Frankfurt am Main, 1982.

Bernhard, Thomas, „Jauregg“, in: Ders., Prosa, Frankfurt am Main, 1971, S. 49-64.

79 Treffend tauchen die Punkte auf im Titel von Simon Critchleys Buch Very Little... Almost Nothing. Death, Philosophy, Literature, London, New York, 1997, 2. erw. Aufl., 2004. Sie unterstreichen die im weiteren Text ausformulierte Forderung nach einer Poetisierung der philosophischen Sprache bzw. nach einer Poetik der Philosophie. 
Blank, Andreas, Literarisierung von Mündlichkeit. Louis-Ferdinand Céline und Raymond Queneau, Tübingen, 1991.

Céline, Louis-Ferdinand, Reise ans Ende der Nacht, übers. v. Hinrich Schmidt-Henkel, Hamburg, 2003.

- Entretiens avec le professeur Y, Paris, 2004.

Critchleys, Simon, Very Little... Almost Nothing. Death, Philosophy, Literature, London, New York, 1997, 2. erw. Aufl., 2004.

Derrida, Jacques, Grammatologie, übers. v. Hans-Jörg Rheinberger, Hanns Zischler, Frankfurt am Main, 1974.

- „Two words for Joyce“, in: Derek Attridge, Daniel Ferrer (Hg.), Post-structuralist Joyce. Essays from the french, übers. v. George Bennington, Cambridge, 1984, S. $145-$ 159.

- Positionen, Gespräche mit Henri Rose, Julia Kristeva u.a., Graz, Wien, 1986.

- „Sporen. Die Stile Nietzsches“, in: Werner Hamacher (Hg.), Nietzsche aus Frankreich, Frankfurt am Main, Berlin, Wien, 1986, S. 129-168.

- Wie nicht sprechen. Verneinungen, übers. v. Hans-Dieter Gondek, Wien, 1989.

- Cosmopolites des tous les pays, encore un effort!, Paris, 1997.

- Demeure. Maurice Blanchot, Paris, 1998.

- Auslassungspunkte. Gespräche, übers. v. Karin Schreiner, Dirk Weissmann, hg. v. Peter Engelmann, Wien, 1998.

- Die Stimme und das Phänomen, Frankfurt am Main, 2003.

- Einsprachigkeit, übers. v. Michael Wetzel, München, 2003.

Frame, Janet, Dem neuen Sommer entgegen, übers. v. Karen Nölle, München, 2010.

Gumbrecht, Hans Ulrich, Stimmungen lesen. Über eine verdeckte Wirklichkeit der Literatur, München, 2011.

Heidegger, Martin, Was ist Metaphysik?, Frankfurt am Main, 2007.

Herder, Johann G., „Ideen“, 7. Buch, in: Ders., Sämtliche Werke, hg. v. Bernhard Suphan, Bd. 13, Berlin, 1877-1913.

Hindus, Milton, Louis-Ferdinand Céline, tel que je l'ai vu, Paris, 1969.

Höchli, Stefan, Zur Geschichte der Interpunktion im Deutschen, Berlin, New York, 1981.

Hohl, Ludwig, Notizen oder Von der unvoreiligen Versöhnung, Notiz 146, Frankfurt am Main, 1981.

Husserl, Edmund, Ideen zu einer reinen Phänomenologie und phänomenologischen Philosophie, Den Haag, 1950.

Joyce, James, Werke, Bd. 6: Briefe II. 1917-1930, übers. v. Kurt Heinrich Hansen, hg. v. Klaus Reichert, Fritz Senn, Frankfurt am Main, 1970.

- Ulysses, London, 2000.

Lethen, Helmut, „,Der Schmerz trägt keine Bedeutung' (Paul Valéry) oder: Gibt es Ereignisse, die den Kulturwissenschaften den Atem verschlagen?", Vortrag in Zürich, 20.5.2011, (Publikation in Vorbereitung).

Mann, Heinrich, Der Untertan, Berlin, 1946.

- „Reichstag“, in: Ders., Macht und Mensch. Essays, Frankfurt am Main, 1989, S. 2631.

Merleau-Ponty, Maurice, Das Sichtbare und das Unsichtbare, übers. v. Regula Giuliani, Bernhard Waldenfels, München, 2004. 
Obermayr, Brigitte, „Auslassungspunkte als Materialspur am Beispiel von A. S. Puskins „Evgenji Onegin““, in: Sonderforschungsbereich 626 (Hg.), Ästhetische Erfahrung. Gegenstände, Konzepte, Geschichtlichkeit, Berlin, 2006.

Pasternack, Gerhard, „Repräsentation und Interpretation“, in: Hans Jörg Sandkühler (Hg.): Welten in Zeichen - Sprache, Perspektivität, Interpretation, Reihe Philosophie und Geschichte der Wissenschaften, Bd. 52, Bern, Berlin, New York, 2002, S. 169192.

Proust, Marcel, Auf der Suche nach der verlorenen Zeit, In Swanns Welt, übers. v. Eva Rechel-Mertens, Frankfurt am Main, 1997.

Rorty, Richard, The Linguistic Turn. Essays in Philosophical Method, Chicago, London, 1992.

Roughley, Alan, Reading Derrida, Reading Joyce, Gainesville, 1999.

Rousseau, Jean-Jacques, „Träumereien eines einsamen Spaziergängers“, in: Ders., Schriften, hg. v. Henning Ritter, Bd. 2, München, 1978, S. 637-760.

Schnitzler, Arthur, Reigen, Frankfurt am Main, 1960.

- „Sterben“, in: Ders., Erzäblungen, Frankfurt am Main, 1992, S. 132-222.

- Ein Liebesreigen. Die Urfassung des „Reigen“, hg. v. Gabriella Rovagnati, Frankfurt am Main, 2004.

Taylor, Charles, Quellen des Selbst, Zur Entstebung der neuzeitlichen Identität, übers. v. Joachim Schulte, Frankfurt am Main, 1994.

- Das Unbehagen an der Moderne, übers. v. Joachim Schulte, Frankfurt am Main, 1995.

Tomasi di Lampedusa, Giuseppe, Der Leopard, übers. v. Charlotte Birnbaum, München, Zürich, 1999.

Wellbery, David E., „Stimmung“, in: Karlheinz Barck u.a. (Hg.), Historisches Wörterbuch ästhetischer Grundbegriffe, Bd. 5, Stuttgart, Weimar, 2003, S. 703-733. 